\title{
Dynamic Decision Models for Staged Software Product Line Configuration
}

\author{
EBRAHIM BAGHERI \\ Ryerson University \\ FAEZEH ENSAN \\ University of British Columbia
}

bagheri@ryerson.ca

\begin{abstract}
Software product line engineering practices offer desirable characteristics such as rapid product development, reduced time-to-market and more affordable development costs as a result of systematic representation of the variabilities of a domain of discourse that leads to methodical reuse of software assets. The development lifecycle of a product line consists of two main phases: domain engineering, which deals with the understanding and formally modeling of the target domain; and application engineering that is concerned with the configuration of a product line into one concrete product based on the preferences and requirements of the stakeholders. The work presented in this paper focuses on the application engineering phase and builds both the theoretical and technological tools to assist the stakeholders in (1) understanding the complex interactions of the features of a product line; (2) eliciting the utility of each feature for the stakeholders and hence exposing the stakeholders' otherwise implicit preferences in a way that they can more easily make decisions; and (3) dynamically building a decision model through interaction with the stakeholders and by considering the structural characteristics of software product line feature models, which will guide the stakeholders through the product configuration process. Initial exploratory empirical experiments that we have performed show that our proposed approach for helping stakeholders understand their feature preferences and its associated staged feature model configuration process is able to positively impact the quality of the end results of the application engineering process within the context of the limited number of participants. In addition, it has been observed that the offered tooling support is able to ease the staged feature model configuration process.
\end{abstract}

Keywords: Software Product Lines, Feature Models, Utility Elicitation, Stakeholder Preferences

\section{Introduction}

Software product lines cater the means for facilitating systematic reuse within the area of software engineering by formally capturing and representing commonalities and variabilities between the applications of the same family [1,2]. Understanding the common and variable aspects of different applications provides insight for reusing common core assets such as services, software components, and modeling artifacts during the software development process. This process often leads to the reduction of development costs due to the fact that software artifacts are shared between different applications and also decrease in time-to-market as applications can be developed more rapidly using existing assets [3]. The concept of product lines has been successfully used in other engineering disciplines such as the automotive and aircraft manufacturing industries. However, the quick evolution and fast-changing-pace of the software development market requires more agile practices for software product lines compared to those in other disciplines.

In order to customize the product line engineering practice for the software development domain, two main phases have been defined in its lifecycle, namely domain engineering and application engineering [3]. The domain engineering phase consists of activities that understand and analyze the requirements of the target domain of interest as a whole; identify the variabilities and the commonalities that may exist 
between the possible applications of that domain; and represent them in a unified formal model. In essence, the outcome of the domain engineering phase should be the representation of most if not all application possibilities for a given domain. Such a representation would among other things constitute the functional characteristics of the applications. Each functional characteristic is referred to as a feature and the set of all features of a domain is referred to as the feature space.

This domain model developed in the domain engineering phase can later be used in the process of developing various instance applications for that domain during application engineering. In application engineering, instance software products are often derived through the consultation with domain stakeholders that have specific requirements in mind. Such requirements will be in turn translated into the restriction and binding of variabilities within the reference software product line architecture and hence into a concrete product [4]. In other words, the preferences and requirements of the stakeholders are taken into account to restrict the feature space by selecting those features that are more desirable and removing those that are of less value. Czarnecki et al. have proposed that the process of selecting the desirable features should be gradually performed with ample interaction with the stakeholders, a process which is referred to as staged configuration [5].

There have already been several interesting work that provide supportive mechanisms and tools for product line designers during the domain engineering phase $[6,7]$. Our focus in this paper is to facilitate the application engineering phase by delivering capabilities to effectively communicate with the stakeholders and understand their needs and preferences. It is clear that the acceptance of a final product developed through the application engineering phase primarily relates to how well it is able to address the needs and concerns of the stakeholders. Therefore, in our view, a successful application engineering process would need to (among others) be able to elegantly exhibit the following characteristics:

- Consider business concerns and non-functional properties: Many domain modeling notations, e.g. feature models, do not consider the explicit representation of non-functional properties such as maintainability and security and also business concerns such as development costs, customer satisfaction and development time [8]. However, both business concerns and non-functional properties play an essential role in the successful uptake of the developed application. Hence, the application engineering process should not only focus on satisfying the functional requirements of the stakeholders, but also effectively factor in these additional considerations at trade-off decision making situations. The application engineering phase should put the non-functional requirements and business concerns of the stakeholders in the context of the non-functional properties of the features, that have already been defined in the domain engineering phase, in such a way that features which more closely resemble the requirements of the stakeholders are selected;

- Support interactive staged configuration: Product line models are most economical when used in large and complex target domains, which can result in software product line domain models that often contain too many interacting features. In light of this, it is not easy to elicit the needs of the stakeholders prior to product line configuration; therefore, the stakeholders need to be engaged in all of the steps of the configuration process, through which different features are considered, their impacts on the final product are evaluated and decisions are gradually made. The interaction can ensure that the stakeholders understand 
the feasible feature space and its limitations and are also comfortable with the design decisions that are made during configuration;

- Reveal impacts of design decisions: The inclusion or exclusion of a feature in/from the feature space can have local impacts in terms of limiting the possibility of selecting other interacting features and global impacts by affecting the quality and desirability of the final product. The stakeholders would need to be able to understand the impact of their design decisions that are gradually made in the staged configuration process. The exposure of the significance of the impacts can help the stakeholders and application engineers to explore different design alternatives before making a final decision;

- Identify significant alternative decision points: As domain models grow in complexity and size, the best points for exploring the feature space may not be very clear for the stakeholders. In other words, the stakeholders can have difficulty in identifying the most critical alternative design decisions that need to be considered at each point in the staged configuration process. For instance, resolving a variation point with many interdependencies in the domain model could potentially lead to a significantly reduced feature space that is easier to explore and hence could be considered as a good candidate for early evaluation. Therefore, it is very desirable to identify significant alternative design decisions for the stakeholders at each point of the configuration process in the application engineering phase; resulting in less complexity and easier decision making.

The work on software product line decision-oriented models [9] deviate from the more traditional feature-oriented approaches [10] where commonalities and variabilities are both captured and represented. Such models view variabilities as decision points; therefore, decision-oriented models representing variability are a formulation of the domain as a decision making plan for product derivation. Decision-oriented models are a significant step forward towards addressing the above characteristics, but they face challenges in terms of predetermined decision points, expensive changeability and lack of adaptability.

In this paper, we propose a staged product line configuration process that centers around the above four characteristics. Our work focuses on feature-oriented software product lines, but dynamically generates decision models during the staged configuration process. In other words, our work does not need a fixed decisionoriented model to be developed in the domain engineering phase and works with existing feature-oriented artifacts such as feature models. We view the product configuration process as a process of stakeholder utility elicitation [11], during which the utility of each feature for the stakeholders is determined. The utility of features is decided through gamble queries [12] where the stakeholders choose between two competing features at a decision point. The series and order of the gamble queries is automatically specified in order to facilitate the utility elicitation process. Therefore, we dynamically generate decision models that adapt based on the choices made at each previous decision point by the stakeholders. The utility elicitation process allows us to create desirability ranking over the existing features, which facilitates feature selection and decision making. Furthermore, our work provides the means to consider the role of non-functional properties and business concerns in the configuration process and propagates feature utility information onto these non-functional aspects of the product line.

The rest of this paper is organized as follows: the next section introduces the basics of feature modeling and belief theory. Section 3 provides an overview of our 

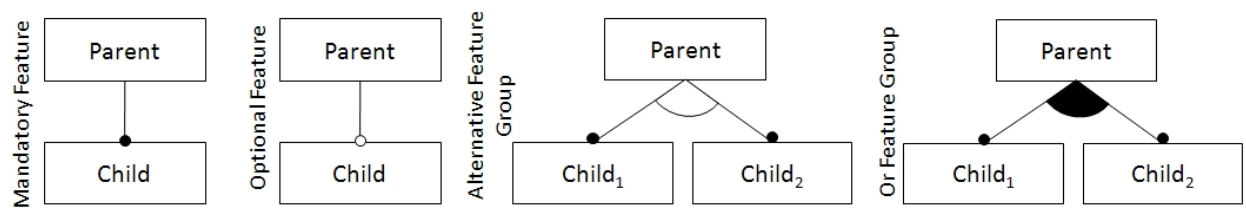

Figure 1. The main graphical notations employed in feature modeling.

proposed staged configuration process and describes how a utility elicitation process can be defined for understanding the preferences of the stakeholders. In Section 4, we show how we extend the feature modeling metamodel to provide support for non-functional properties and business concerns. The details of our proposed work are discussed in Section 5, which is followed by a discussion on the tooling support in Section 6. The setup and the structure of our exploratory empirical experiments is presented in Section 7, which is followed by the explanation of the observed outcomes in Section 8. Before concluding the paper, a discussion including the limitations of our work and the lessons learnt, and also a review of the related work are presented.

\section{Preliminaries}

In this section, the overview of feature modeling and belief theory is outlined in a way that the paper is self-contained.

\subsection{Feature Models}

Features are important distinguishing aspects, qualities, or characteristics of a family of systems $[13,10]$. They are widely used for depicting the shared structure and behavior of a set of similar systems. To form a product family, all the various features of a set of similar/related systems are composed into a feature model. Feature models can be represented both formally and graphically; however, the graphical notation depicted through a tree structure is more favored due to its visual appeal and easier understanding. More specifically, graphical feature models are in the form of a tree whose root node represents a domain concept, e.g., a domain application, and the other nodes and leafs illustrate the features. In this context, a feature is a concept property related to a user-visible functional or non-functional requirement, e.g., domain application task, modeled in a way to capture commonalities or possibly differentiate among product family variants [14].

Figure 1 depicts the graphical notation of the feature relationships. The tree structure of feature models falls short at fully representing the complete set of mutual interdependencies of features; therefore, additional constraints are often added to feature models and are referred to as Integrity Constraints (IC).

The Graph Product Line (GPL) is a widely used feature model, [15] shown in Figure 2, which covers the classical set of applications of graphs in the domain of Computer Science. The intention is to be able to develop configurations of GPL, which are able to address different problem domains. For instance, GPL can be configured to perform several graph search algorithms over a directed or undirected 


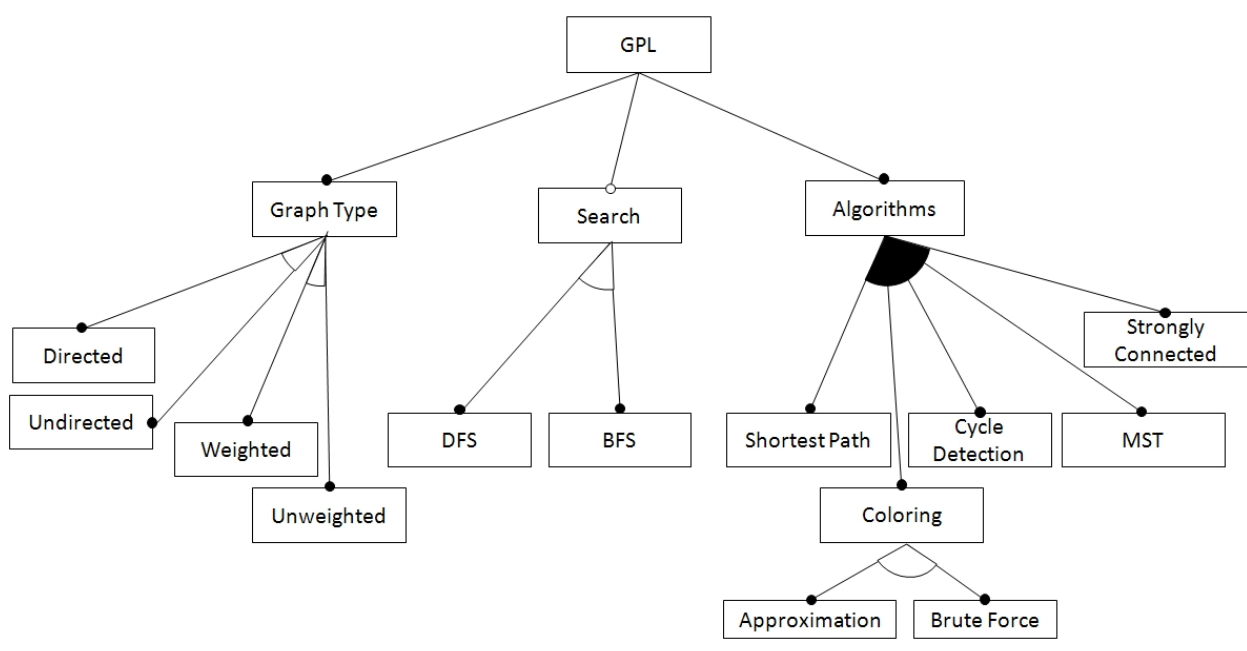

Figure 2. The graph product line feature model.

graph structure. As it can be seen, GPL consists of three main features: 1) Graph Type: features for defining the structural representation of a graph; 2) Search: traversal algorithms in the form of features that allow for the navigation of a graph; and 3) Algorithms: other useful algorithms that manipulate or analyze a given graph. Clearly, not all possible configurations of the features of GPL produce valid graph programs. For instance, a configuration of GPL that checks if a graph is strongly connected cannot be implemented on an undirected graph structure. Such restrictions are expressed in the form of integrity constraints. These integrity constraints ensure the correct composition of features in the various final software products developed from this feature model.

\subsection{Belief Theory}

Belief theory is one of the theoretical models that is able to numerically quantify uncertainty in human knowledge in an effective manner [16]. It is a useful tool for the evaluation of the information gathered by the involvement of human experts. Dempster-Shafer (DS) theory of evidence is amongst the most widely employed models of belief theory that provides appropriate means for approximate and collective reasoning under uncertainty $[17,18]$. It is an extension to the traditional probability theory where probabilities are assigned to sets (or intervals) as opposed to singleton variables [19].

The foundation of the DS theory of evidence is based on a set of hypotheses $\theta$ called the frame of discernment defined as:

$$
\theta=\left\{H_{1}, H_{2}, \ldots, H_{N}\right\} .
$$

The frame of discernment consists of $\mathrm{N}$ exclusive hypotheses. Based on the frame of discernment $\theta$, the powerset $2^{\theta}$ composed of all the possible propositions can be created such that:

$$
2^{\theta}=\left\{\phi,\left\{H_{1}\right\},\left\{H_{2}\right\}, \ldots,\left\{H_{N}\right\},\left\{H_{1} \cup H_{2}\right\}, \ldots, \theta\right\} .
$$


The base function required for the DS theory of evidence is a basic belief assignment, which is similar to probability distribution, but differs in the fact that belief masses are distributed over the elements of the powerset $2^{\theta}$ and not only on the singleton elements of $\theta$; therefore, composite subsets of the powerset can also receive a degree of the belief mass. In other words, the truthful subsets of the powerset can receive a degree of belief mass; therefore, the belief mass assigned to an atomic set such as $\psi \in 2^{\theta}$ is taken as the belief that the given set is true. The belief in $\psi$ is interpreted as the absolute faith in the truthfulness of $\psi$, which not only relies on the belief mass assigned to $\psi$, but also to belief masses assigned to subsets of $\psi$.

Definition 1 A belief mass assignment is a mapping $m: 2^{\theta} \rightarrow[0,1]$ that assigns $m_{\theta}(\psi)$ to each subset $\psi \in 2^{\theta}$ such that:

(1) $m_{\theta}(\psi) \geq 0$,

(2) $m_{\theta}(\varnothing)=0$,

(3) $\sum_{\psi \in 2^{\theta}} m_{\theta}(\psi)=1$.

$m_{\theta}(\psi)$ is then called the belief mass of $\psi$. A belief mass assignment is called dogmatic if $m_{\theta}(\theta)=0$, since all the possible belief masses have been spent on the subsets of $\theta$.

Definition $2 A$ belief function corresponding with $m_{\theta}$, a belief mass assignment on $\theta$, is a function $b: 2^{\theta} \rightarrow[0,1]$ defined as:

$$
b(\psi)=\sum_{\varphi \subseteq \psi} m_{\theta}(\varphi), \quad \varphi, \psi \in 2^{\theta} .
$$

Analogously, disbelief is the total belief that a set is not true.

Definition $3 A$ disbelief function corresponding with $m_{\theta}$, a belief mass assignment on $\theta$, is a function $d: 2^{\theta} \rightarrow[0,1]$ defined as:

$$
d(\psi)=\sum_{\varphi \cap \psi=\varnothing} m_{\theta}(\varphi), \quad \varphi, \psi \in 2^{\theta} .
$$

In cases where $b(\psi)+d(\psi)=1$, DS theory can be considered to be reduced to probability theory. To address the degree of uncertainty which is inherent in the above definitions, Jøsang provides a complementary definition, uncertainty, which computes the degree of possible confusion in the belief assignment [20].

Definition 4 An uncertainty function corresponding with $m_{\theta}$, a belief mass assignment on $\theta$, is a function $u: 2^{\theta} \rightarrow[0,1]$ defined as:

$$
u(\psi)=\sum_{\varphi \cap \psi \neq \varnothing, \varphi \nsubseteq \psi} m_{\theta}(\varphi), \quad \varphi, \psi \in 2^{\theta} .
$$

With the use of the above definitions, Subjective logic [20] extends DempsterShafer theory of evidence. A belief expression in Subjective logic is defined as a triple $\chi_{x}^{A}=\left(b_{x}^{A}, d_{x}^{A}, u_{x}^{A}\right)$ also known as the opinion of an expert $A$ regarding hypothesis $x\left(\chi_{x}^{A}\right)$. So for instance, $(1,0,0)$ and $(0,0,1)$ are two subjective opinions that 
represent complete belief and complete uncertainty regarding a given phenomenon. It can be shown with this definition that belief $\left(b_{x}^{A}\right)$, disbelief $\left(d_{x}^{A}\right)$, and uncertainty $\left(u_{x}^{A}\right)$ elements of an opinion should satisfy:

$$
b_{x}^{A}+d_{x}^{A}+u_{x}^{A}=1 .
$$

Subjective logic defines a large number of operators that allow the manipulation of subjective opinions. In this paper, we employ two main operators, namely discounting and consensus [20].

Discounting is an operator that given an opinion of a specific agent with regards to a phenomenon, and the opinion of a second agent about the first agent, it is able to estimate the opinion of the second agent about the phenomenon of interest. Therefore, it is possible to develop an idea about a phenomenon that has only been indirectly observed by a third party given the reliability assigned to that third party. So, if the third party is completely reliable, his reports of the observation of the phenomenon will be acceptable; but, as the lack of trust in that third party's observation increases, the uncertainty with regards to his observations increase as well. Its important to notice that reports provided by an agent that is not trusted cannot be directly attributed to disbelief but rather should be assumed as uncertain. In other words, one can only be uncertain of the truthfulness of the information provided by an unreliable source but cannot directly infer disbelief in those information.

Definition 5 Let $\chi_{x}^{A}=\left(b_{x}^{A}, d_{x}^{A}, u_{x}^{A}\right)$, and $\chi_{A}^{B}=\left(b_{A}^{B}, d_{A}^{B}, u_{A}^{B}\right)$ be two subjective opinions. The discounted opinion is calculated as:

$$
\chi_{x}^{B A}=\left(b_{A}^{B} b_{x}^{A}, b_{A}^{B} d_{x}^{A}, d_{A}^{B}+u_{A}^{B}+b_{A}^{B} u_{x}^{A}\right) .
$$

The second operator that will be employed is the consensus operator. The intention of the consensus operator is to meaningfully summarize a corpus of data elements coming from various sources in order to develop a common unification between the beliefs of the participating sources. In other words, if multiple observation reports are received from different agents regarding the same phenomenon, the consensus operator will allow us to combine the information into one representative summary in order to form an opinion based on these collected information.

Definition 6 Let $\chi_{x}^{A}=\left(b_{x}^{A}, d_{x}^{A}, u_{x}^{A}\right)$ and $\chi_{x}^{B}=\left(b_{x}^{B}, d_{x}^{B}, u_{x}^{B}\right)$ be two opinions about a common fact $x$ stated by two different information sources $A$ and $B$, and let $\kappa=u_{x}^{A}+u_{x}^{B}-u_{x}^{A} u_{x}^{B}$. When $u_{x}^{B} \rightarrow 0$, and $u_{x}^{A} \rightarrow 0$, the relative dogmatism between the two opinions are defined using $\gamma=u_{x}^{B} / u_{x}^{A}$. Now $\chi_{x}^{A, B}=\left(b_{x}^{A, B}, d_{x}^{A, B}, u_{x}^{A, B}\right)$ is a fair representative of both opinions, Consensus operator $\oplus$ outcome, such that:

\begin{tabular}{ll} 
when $\kappa \neq 0$ & when $\kappa=0$ \\
\cline { 3 - 3 } & \\
$b_{x}^{A, B}=\left(b_{x}^{A} u_{x}^{B}+b_{x}^{B} u_{x}^{A}\right) / \kappa$ & $b_{x}^{A, B}=\frac{\gamma b_{x}^{A}+b_{x}^{B}}{\gamma+1}$ \\
$d_{x}^{A, B}=\left(d_{x}^{A} u_{x}^{B}+d_{x}^{B} u_{x}^{A}\right) / \kappa$ & $d_{x}^{A, B}=\frac{\gamma d_{x}^{A}+d_{x}^{B}}{\gamma+1}$ \\
$u_{x}^{A, B}=\left(u_{x}^{A} u_{x}^{B}\right) / \kappa$ & $u_{x}^{A, B}=0$ \\
\hline
\end{tabular}

The Consensus operator has been shown to have a stable behavior under various conditions and even while merging conflicting dogmatic beliefs [21]. It satisfies 
two important algebraic properties, i.e., commutativity $(A \oplus B=B \oplus A)$, and associativity $(A \oplus[B \oplus C]=[A \oplus B] \oplus C)$, which are of great significance while merging peer information sources.

Finally, similar to probability theory where the probability space can be connected to the evidence space through counting the number of positive and negative observations, it is possible to offer such a mapping between the belief space and the evidence space in Subjective logic. Given observations for phenomenon $x$, the mapping is as follows:

$$
\begin{aligned}
b_{x} & =\frac{r}{r+s+1} \\
d_{x} & =\frac{s}{r+s+1} \\
u_{x} & =\frac{1}{r+s+1}
\end{aligned}
$$

where $r$ and $s$ are the number of positive and negative observations, respectively. The interesting point about this mapping is as more observations are made about a phenomenon, the degree of uncertainty will decrease. We will benefit from Subjective logic in order to capture the utility of the features of a feature model for the stakeholders in the application engineering configuration process.

\section{Approach Overview}

The goal of our work is to develop a mechanism that would engage with software stakeholders and identify their preferences in the context of the software features available in a software product line. In other words, the stakeholders would be able to identify the utility of each feature of the product line by interacting with this mechanism in the application engineering configuration process. The overview of the steps of our approach is shown in Figure 3. The initial input of our approach is a fully developed feature model that needs to be configured and the gradual inputs are the stakeholders' feedbacks on the queries made by our approach. The output of our approach is the preference of the stakeholders over the available features in the feature model and also their preference over non-functional properties and business concerns. As seen in the figure, our approach consists of seven major steps:

1. The first step involves the identification of the possible decision points in the decision path. Decision points are points of variability in the product line where the stakeholders would need to select from amongst the available alternatives. Besides the explicit variability points, other features that have some form of interaction with variable features could also be considered as parts of a decision point. The outcome of this step is the identification of the decision points;

2. Once the decision points have been identified, the second step develops a ranking over them based on a measure referred to as expected utility. This measure shows how much more information the stakeholder would attain if she makes a decision with regards to a given variability point. Therefore, decision points that have higher expected utilities would be better candidates to be handled earlier;

3. The decision points are then ranked descendingly based on their expected utility. In this ranking, the decision point that is ranked highest has the most likelihood of revealing more useful information for the stakeholders; 


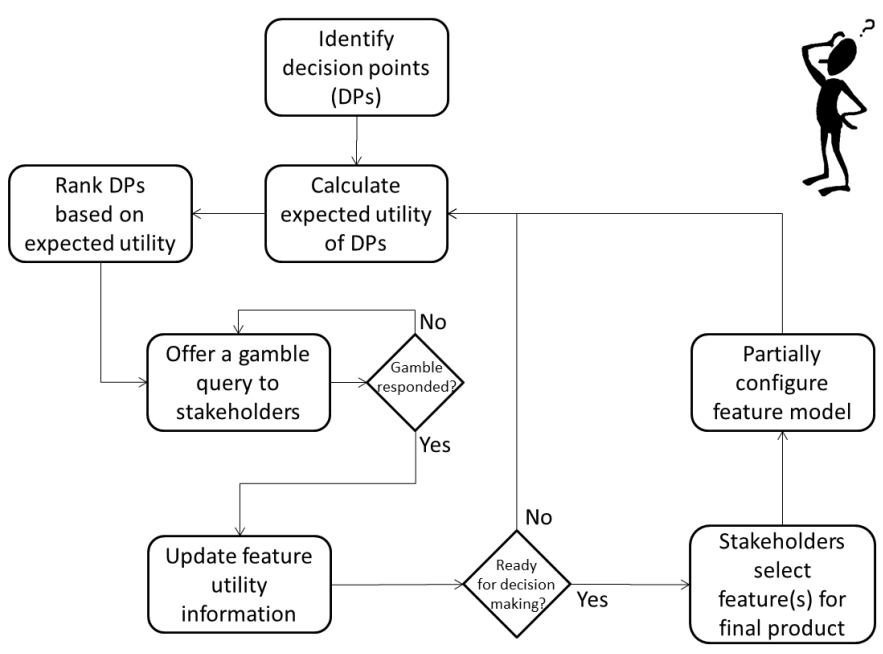

Figure 3. Overview of our approach.

4. In the fourth step, the decision point with the maximum expected utility will be presented to the stakeholders in the form of a gamble query. The stakeholders would need to decide between the two competing offers that are provided to them in the gamble. They would also have the chance to ignore a gamble query and request the next query to be presented to them. The choice of either of the two options in the gamble query would not only reveal the utility of the selected option but also the utility of other features that are related to that feature or the feature not selected in the gamble;

5. Once the stakeholders make a selection in a gamble query, it is possible to update the utility of the related features in the feature model. The feature that was selected in the gamble and its related features will receive a positive impact on their utility while the feature that was not selected and its associated features will be negatively impacted. Furthermore, given that features can be associated with abstract concerns, the impacts on the utility of a feature will also be propagated to its attributed abstract concerns as well.

6. In the fifth step, the stakeholders will receive direct feedback in terms of the updated utility information of the features and abstract concerns. This includes the ranking of the most desirable features to be selected in the configuration process and also the ranking of the abstract concerns. It should be noted that this feedback is developed based on the utility elicitation process until that point and will be updated accordingly as more gamble queries are answered;

7. At this stage, the stakeholders will have the option to look at the utility information of the available features and decide whether they would like to select one or more features to be included in the final product or not. If they choose to select one or more features, variability will be removed from the feature model, the feature space will be restricted and the process is repeated by continuing from Step 2 with the restricted feature model. However, if no features are selected at this point, this process is repeated by returning to the second step with the unchanged feature model where the new expected utility of the deci- 
sion points are recalculated based on the information gained from the answered gamble query.

It is noteworthy that the choices made in the gamble queries are different from feature selections for the configuration process. The choices made in the gamble queries only represent the preference of the stakeholders; whereas, the stakeholders would have the option to choose a different feature (even a feature that they did no choose in a gamble) for model configuration once they receive an overall feedback on how their preferences impact the utility of other features in the model. Therefore, the features selected in the gambles do not necessarily need to be selected for inclusion in the final product.

\section{Extending Feature Modeling Notation}

For our work, we have extended the abstract syntax metamodel of the cardinalitybased feature modeling notation [22] in a way that it provides support for some additional key concepts in our work, namely abstract concern and utility. The concept of abstract concerns refers to either non-functional properties or business concerns that are important for the stakeholders. As shown in Figure 4, each feature or feature group can be annotated with any number of abstract concerns in order to show that the feature (group) has some form of impact on those specific abstract concerns. For instance, a feature such as brute force from Figure 2 can be annotated with the execution time abstract concern. This shows that the brute force feature impacts the execution time of any application that contains it which can be a concern for the stakeholders. The linkage between features and abstract concerns is developed using the utility concept. Now, it is important to be able to show whether the impact between a feature and an abstract concern is positive or negative and what is the significance of the impact. In order to show whether brute force has positive or negative impact on execution time, we have included a property for the utility concept, i.e. impact, that allows us to represent the polarity and significance of the effect of a feature on an abstract concern. The impact property has been defined as a Subjective logic opinion, which allows us to both capture polarity in terms of belief and disbelief (positive or negative impact) and also significance through the amount of belief mass assigned to either belief or disbelief. So, it is now possible to say that brute force negatively impacts execution time by simply using the subjective logic opinion of $\left(b_{x}, d_{x}, u_{x}\right)=(0,1,0)$. Our interpretation of this statement is given that all the belief mass is assigned to disbelief $\left(d_{x}\right)$; therefore, the feature can be seen as having negative impact on the abstract concern. If the belief mass was assigned to belief in the opinion, then we would understand that the feature has a positive impact on the abstract concern. There are two desirable aspects to such a representation: 1) the ability to represent varying degrees of impact: by assigning different amounts of belief mass to either belief or disbelief, we are able to show how much influence a feature has on a given abstract concern. For instance, a small amount of belief mass assigned to the belief dimension would show that although the feature has positive impact on the abstract concern, the impact is not significant; 2) the representation of neutral or uncertain impacts: in this case by assigning the belief mass to the uncertainty dimension of the subjective opinion, we are able to show that either the feature and the abstract concern do not have any interaction with each other or that we are not aware of such interaction. 


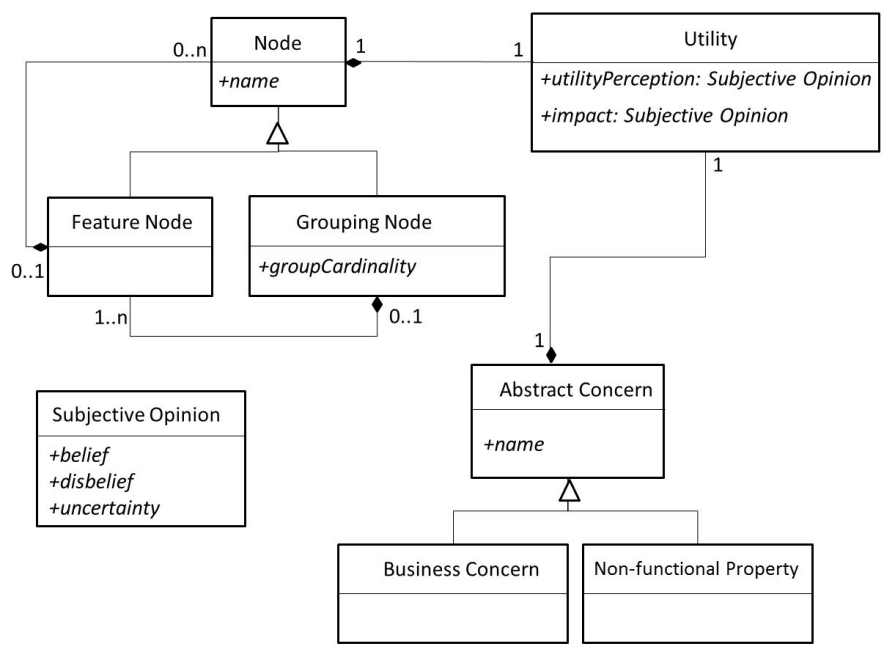

Figure 4. The extended abstract syntax metamodel for feature models.

Table 1. The linguistic values used for representing impact of features on abstract concerns.

\begin{tabular}{lccccc}
\hline Linguistic Term & Very Positive & Positive & Neutral & Negative & Very Negative \\
\hline Subjective Opinion & $(1,0,0)$ & $(0.75,0,0.25)$ & $(0,0,1)$ & $(0,0.75,0.25)$ & $(0,1,0)$
\end{tabular}

The main issue with the use of Subjective opinions with human subjects is that it is hard for humans to come up with accurate belief masses to be assigned to each of the dimensions of the Subjective belief opinion. Researchers have already proposed ways to deal with this issue including the use of linguistic variables $[23,24,25]$. In this way, the human subjects will only be interacting with understandable linguistic expressions that would be later automatically translated into Subjective opinions to be used in the underlying inference processes. As will be shown in the tool support section of this paper, we have used a similar approach whereby the domain engineers are able to use linguistic variables to denote the impact of features on abstract concerns. We provide five linguistic terms to the domain engineers that can be easily converted into Subjective opinions. These linguistic terms and their corresponding Subjective opinions are shown in Table 1. Hence, the statement, brute force has a very negative impact on execution time can automatically be converted into $(0,0,1)$ as the Subjective opinion.

The second property that we have introduced into the utility concept is utilityPerception. This property allows us to capture the utility of each feature for the stakeholders. In other words, utilityPerception shows how desirable or useful a feature is for the stakeholders. Unlike the impact property that needs to be directly manipulated by the stakeholders during the domain engineering phase, the utilityPerception property will only be automatically updated during the application engineering phase based on the results of the gamble queries and the choices made by the stakeholders. This value will be used to calculate the desirability of features for the stakeholders and is constantly updated as new information is received from the stakeholders through answering gamble queries. The utilityPerception property is again of Subjective opinion type; therefore, a feature annotated with $(0,0,1)$ shows that the utility of the feature for the stakeholders is still unknown, 
while another feature annotated with $(0,1,0)$ is known to be highly undesirable for the stakeholders. Similarly, varying amounts of belief mass assigned to belief or disbelief allows for the representation of a utility spectrum.

In summary, the extended feature model notation allows us to both capture and represent abstract concerns (non-functional properties and business concerns) and feature utility information. The varying degrees of each of these concepts are represented using Subjective logic opinions.

\section{Feature Utility Elicitation Details}

We view the whole process of staged configuration as a utility elicitation process during which the preferences of the stakeholders over the existing features are identified. The features with higher utility for the stakeholders will be the ones to be selected to be included in the product-to-be. In this section, we will explain the technical details of how feature utilities are identified through asking gamble queries from the stakeholders during the staged configuration process.

\subsection{Gamble Query Structure}

In essence, gamble queries are a means to differentiate between the desirability of two competing features. A gamble query allows the stakeholder to carefully consider two competing features and decide which one between the two is more desirable. A gamble query consists of two features that cannot co-exist in a final configuration; therefore, the stakeholders would need to make a choice between either of these two features. For instance in Figure 2, the approximation and brute force features are two competing features that cannot be present in the same product simultaneously. A gamble query would ask the stakeholders the following question: if you had the option to select from one of the following two features, which one would be a more desirable choice? In addition to the functional aspect of the gamble query, the stakeholders will be provided with information regarding the relevant abstract concerns related to each feature. Hence, the stakeholders will be making a choice between two functional features in light of additional abstract concerns.

For instance, an actual gamble query would be similar to the following: If you had the option to select from one of the following two features, which one would be a more desirable choice?

Option 1 -approximation: Positive on execution time; Very Negative on precision; Negative on implementation costs.

Option 2 -brute force: Very Negative on execution time; Very Positive on precision; Positive on implementation costs.

In this example, the stakeholders will understand that although the approximation feature will provide benefits in terms of execution time but it will negatively impact the other two abstract concerns. On the other hand, while brute force is not so effective regarding execution time but it offers good impact on the other abstract concerns. The presentation of the abstract concerns in a gamble query allows the stakeholders to understand the impact of the selection of certain functional features on non-functional properties and business concerns. Hence, from a broader 
perspective, the intention of a gamble query is not only to elicit the desirability of functional features but to also understand the appeal of the abstract concerns for the stakeholders.

\subsection{Gamble Utility Assessment}

The stakeholders' opinion regarding the options of a gamble query is not necessarily deterministic, i.e., the stakeholders would not always be prepared to select one option over the other. Their opinion can be anywhere in the range from highly prefer option 1 to highly prefer option 2 . For this reason, we allow the stakeholders to answer gamble queries by selecting a value from this range whose middle point is the indifference between the two options. The stakeholders will be able to select from the following: somewhat prefer, prefer and highly prefer one of the options or choose to remain indifferent (neutral). For example, the stakeholders could specify that they highly prefer the brute force option over approximation or alternatively to say that they somewhat prefer approximation over brute force.

Once feedback is provided by the stakeholders regarding a gamble query, it is possible to update the utility of both features present in the gamble (Step 5 of Figure 3). Given that the utilityPerception property is a Subjective opinion, we are able to use the technique introduced in the preliminaries section to update its value. We benefit from the mapping between evidence space and belief space to update the utility of a feature. In other words, the utilityPerception related to each feature is updated as positive and negative evidence for that feature are observed in gamble queries. Given that we have allowed the stakeholders to select from indifference (0), somewhat prefer (1), prefer (2) and highly prefer (3) for one of the options, we are able to consider this as a weighted positive observation for the selected feature and weighted negative observation for the feature not selected. Here for calculating the weighted observation, somewhat prefer receives the lowest weight equal to one, prefer receives the weight of two and highly prefer is assigned the highest weight equal to three. For instance, for the above example mentioning that the stakeholder somewhat prefers approximation over brute force, we assume that we have made one positive observation for approximation and one negative observation for brute force. Similarly, highly prefering brute force over approximation would entail three positive observations in favor of brute force and three accounts of negative observation against approximation.

Based on the weighted observations, each gamble query is able to produce two new Subjective opinions regarding the features present in the gamble. Let us refer to the winning feature of the gamble as $f_{w}$ and the loosing feature as $f_{l}$. The new opinions formed regarding the utility of $f_{w}$ and $f_{l}$ can be calculated using Equations 8-10. The new subjective opinion about the utility of $f_{w}, \mathbb{U}^{n}\left(f_{w}\right)$, is computed by the weighted positive observation as $r$ and no negative weighted observations for $s$. Likewise, the new subjective opinion about the utility of $f_{l}, \mathbb{U}^{n}\left(f_{l}\right)$, would be the weighted negative observation as $s$ and no positive observations for $r$. Both $\mathbb{U}^{n}\left(f_{w}\right)$ and $\mathbb{U}^{n}\left(f_{l}\right)$ represent the utility of $f_{w}$ and $f_{l}$ by observing the outcome of the gamble. This could be considered as the utility for these two features if no prior utility information for the features existed. However, if the features already possess some utility information gained through prior gambles, it is not possible to discard prior utility information and simply replace them with the new utility information. 
For this reason, we employ a utility update strategy where prior utility information for each feature is updated once new utility information becomes available.

Definition 7 Let $\mathbb{U}(f)$ be the current utility information of feature $f$ and $\mathbb{U}^{n}(f)$ be the new subjective opinion about the utility of that feature, the updated utility for $f$ is calculated as follows:

$$
\mathbb{U}^{*}(f)=\mathbb{U}(f) \oplus \mathbb{U}^{n}(f)
$$

where $\oplus$ denotes the Subjective logic consensus operator.

The utility update strategy takes both utility information into account and develops an aggregate opinion regarding the utility of the feature based on both current and prior gambles. It is worth noting that a feature with no prior utility information is associated with a completely uncertain perceivedUtility, which shows that no information regarding its utility is available. Under such circumstance, $\mathbb{U}(f)$ is equal to $(0,0,1)$, in which case the consensus operator will act as an identity function and returns $\mathbb{U}^{n}(f)$ as the updated utility.

Example 1 Assume that in a gamble query the stakeholders have stated that they highly prefer approximation $\left(f_{w}\right)$ over brute force $\left(f_{l}\right)$. Further suppose that prior utility information for each of the features is $\mathbb{U}\left(f_{w}\right)=(0.5,0,0.5)$ and $\mathbb{U}\left(f_{l}\right)=$ $(0,0,1)$. Given the result of the gamble, the new utility for the features would be $\mathbb{U}^{n}\left(f_{w}\right)=\left(\frac{3}{3+0+1}, \frac{0}{3+0+1}, \frac{1}{3+0+1}\right)$ and $\mathbb{U}^{n}\left(f_{l}\right)=\left(\frac{0}{3+0+1}, \frac{3}{3+0+1}, \frac{1}{3+0+1}\right)$. Therefore, the updated utility information for each of these features would be calculated based on Definition 7 by computing $\mathbb{U}^{*}\left(f_{w}\right)=\mathbb{U}^{n}\left(f_{w}\right) \oplus \mathbb{U}\left(f_{w}\right)$ and $\mathbb{U}^{*}\left(f_{l}\right)=\mathbb{U}^{n}\left(f_{l}\right) \oplus$ $\mathbb{U}\left(f_{l}\right)$, the final result of which are as follows: $\mathbb{U}^{*}\left(f_{w}\right)=(0.8,0,0.2)$ and $\mathbb{U}^{*}\left(f_{l}\right)=$ $(0,0.75,0.25)$; showing that approximation has a high utility value while brute force is not desirable from the perspective of the stakeholders.

\subsection{Utility Propagation}

As discussed above, the outcome of a gamble query would enable us to update the utility of the two features that were involved in a gamble (Step 5 of Figure 3). However, even more information can be inferred from a gamble query given the hierarchical nature of feature models and the internal feature interactions. We propose that it is possible to propagate the updated utility information of the features in a gamble throughout the rest of the feature model using two mechanisms, namely propagation through feature interaction and propagation through abstract concerns.

5.3.1. Propagation through feature interaction From amongst the various types of feature interaction in a feature model, there are three main types of interaction that form the basis for utility interdependence between different features:

- Hierarchical parent-child relations - The utilities of parent and child features can have close connection with each other. From the perspective of this feature interaction type, if the utility of the parent is high (low) for the stakeholders, then there is a high probability that the utility of the child feature is also high (low). The reverse is also true. This interdependence does not always hold given that other child features may also exist that impact the utility of the parent; 
however, each feature has its own share of impact on the parent (children). Therefore, it is possible to draw conclusions regarding the utility of a feature based on the utility of its children (parent).

- Sibling 'and' relations - Sibling features that are connected to each other with an and relation are required to appear together in a final product configuration if either of the features are selected. Therefore, the utility of each sibling feature in this type of interaction can be considered to be dependant on the others in a way that the utility of a feature can proportionally change with an increase or decrease in the utility of the other siblings.

- Integrity constraints - Depending on the type of the constraint, the features of an integrity constraint can have direct or reverse impacts on each others' utility. An includes constraint acts similar to a sibling 'and' relation and hence the features with such a constraint can have direct impact on each others' utility. On the other hand, an excludes constraint acts in the opposite way and hence the features in this type of constraint can have reverse impact on each others' utility.

Figure 5 shows these different forms of utility propagation based on feature interaction. The main idea behind utility propagation is to infer partial utility information for features not observed in the gamble by only relying on their interactions with the features that participated in a gamble. So for instance, if a feature such as $f_{1}$ has an includes relation with a feature $g$ that has been chosen in a gamble, we can with some reasonable possibility also infer that given the utility of $g_{1}$ has increased that the utility of $f_{1}$ could also be increased. Similarly, if two other features such as $f_{2}$ and $g_{2}$ have an excludes relation and $g_{2}$ has been selected in a gamble, it is possible to infer that the utility of $f_{2}$ would decrease.

In light of the fact that feature interaction is only one of the contributing factors to the utility of a feature, the utility propagation strategy that we propose employs a decay factor to lessen the impact of utility propagation and only partially impact the utility of unobserved interacting features. Algorithm 1 shows the details of the utility propagation method based on feature interactions. As seen on lines 1 and 2 of the algorithm, the updated utility information for feature $f\left(\mathbb{U}^{*}(f)\right)$ has been decayed using the Subject logic discount operator $(\otimes)$ in order to reduce the impact of the updated utility of $f$ on interacting features. The two decaying factors, i.e., direct decay beta and reverse decay $\beta^{-}$, increase the uncertainty in $\mathbb{U}^{*}(f)$ before it is propagated onto other features. The decayed utility information that is propagated is either $\mathbb{U}_{d}^{*}(f)$ or $\mathbb{U}_{r d}^{*}(f)$ depending on direct or reverse impact of $f$ on the interacting feature, which are Subjective opinions with lower belief or disbelief values and higher uncertainty compared to $\mathbb{U}^{*}(f)$. The reason that the utility of $f$ is discounted to increase uncertainty is that there may be other contributing factors to the utility of the other features other than the utility of $f$, which are not known in the propagation process that need to be attributed to the uncertainty dimension of the propagated utility.

In order to propagate the updated utility of feature $f$ onto its parent, lines 3-6 have been instructed. On line 4 , the utility of the parent is combined using the Subjective consensus operator with the discounted version of the updated utility of $f$ to form the new utility of the parent feature. Once the utility of the parent feature is updated, it needs to also be propagated recursively as shown on line 6 . In order to make sure that this process does not fall into a infinite loop, we only allow 


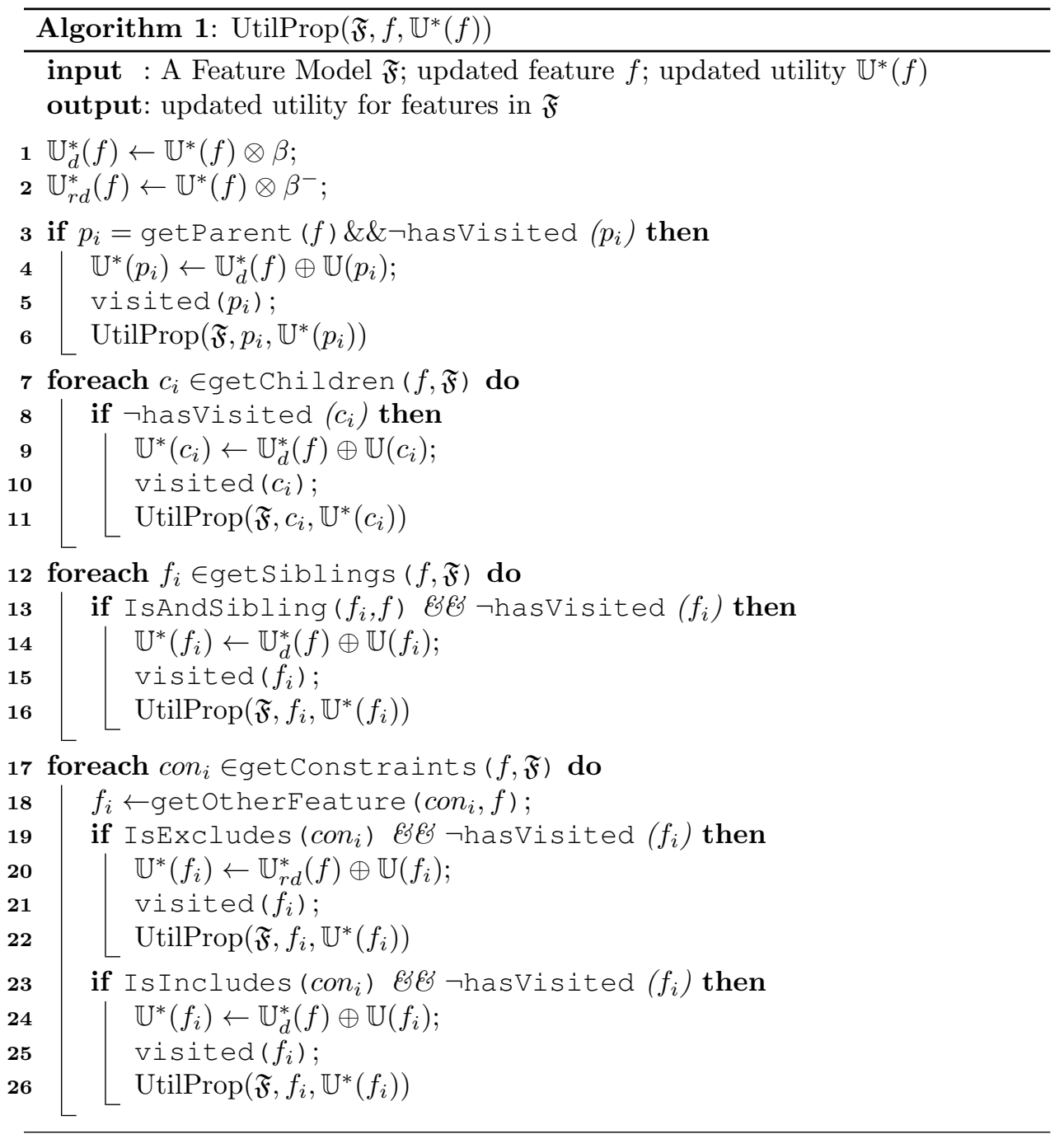

a one-time propagation of utility to each feature for every single gamble and hence the tracking of feature visits on line 5. The rest of the algorithm follows a similar recursive utility propagation pattern for child features, 'and' siblings and integrity constraints. It should be noted that the only place where $\mathbb{U}_{r d}^{*}(f)$ is employed is on line 20 due to the fact that when two features are interacting based on an excludes integrity constraint, the reverse decayed form of the utility information should be passed onto the interacting feature to model the reverse impact of the two features on each other.

The propagation of utility information based on Algorithm 1 allows us to develop partial utility information for features that have not been observed in gambles. This could potentially lead to the benefit of answering less gamble queries by the stakeholders.

Example 2 Lets suppose that Figure 6 represents a part of a feature model in which feature $f_{3}$ has been involved in a gamble and been chosen in the gamble as the more desirable feature. Now based on the outcome of the gamble, utility of 

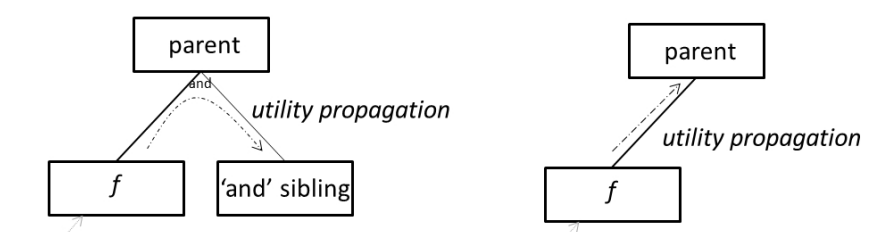

$\mathbb{U}^{*}(f)$

$\mathbb{U}^{*}(f)$

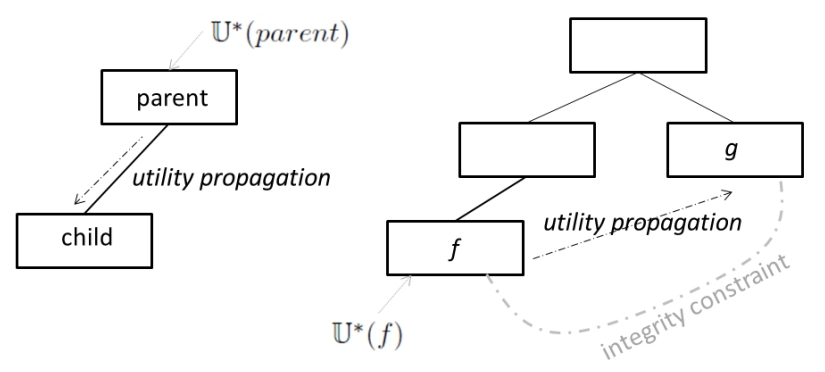

Figure 5. Various forms of utility propagation based on feature interaction.

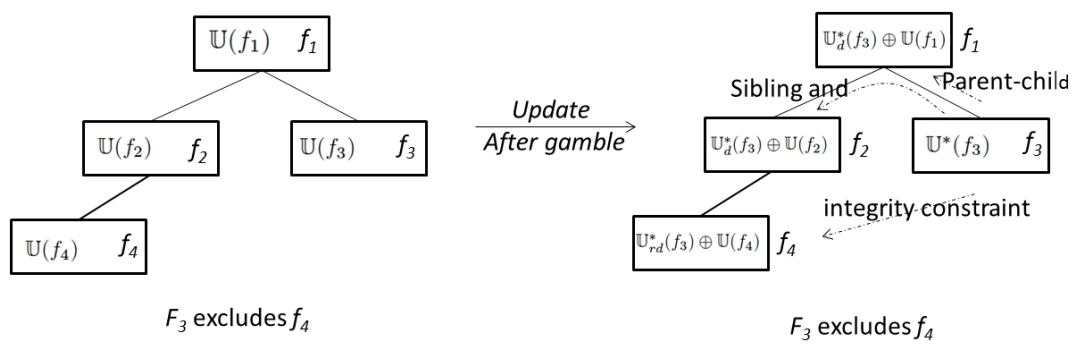

Figure 6. Example utility propagation through feature interaction.

$f_{3}$ will be updated into $\mathbb{U}^{*}\left(f_{3}\right)$. Furthermore, the rest of the interacting features with $f_{3}$ will need to be updated as well (also recursively with other features that are connected to these interacting features). Feature $f_{1}$ has a parent-child relation with $f_{3}$ and $f_{2}$ is a sibling of $f_{3}$ with an 'and' relation. For these two features, it is possible to update their utility with a direct decayed utility from $f_{3}$. However, feature $f_{4}$ is related to $f_{3}$ through an excludes integrity constraint. Therefore, the utility of $f_{4}$ will need to be updated using reverse decayed utility of $f_{3}$.

5.3.2. Propagation through abstract concerns The second form of utility propagation that we propose is based on the premise that features impacting the same abstract concern can to some restricted extent have impact on each others' utility. For instance, if both features $f$ and $g$ positively impact the same abstract concern $a c$ and feature $f$ has been selected in a gamble, it could be inferred that given the desirability of feature $f$, abstract concern $a c$ is also desirable for the stakeholders. As an indirect inference, one could also conclude that feature $g$ is also partially 


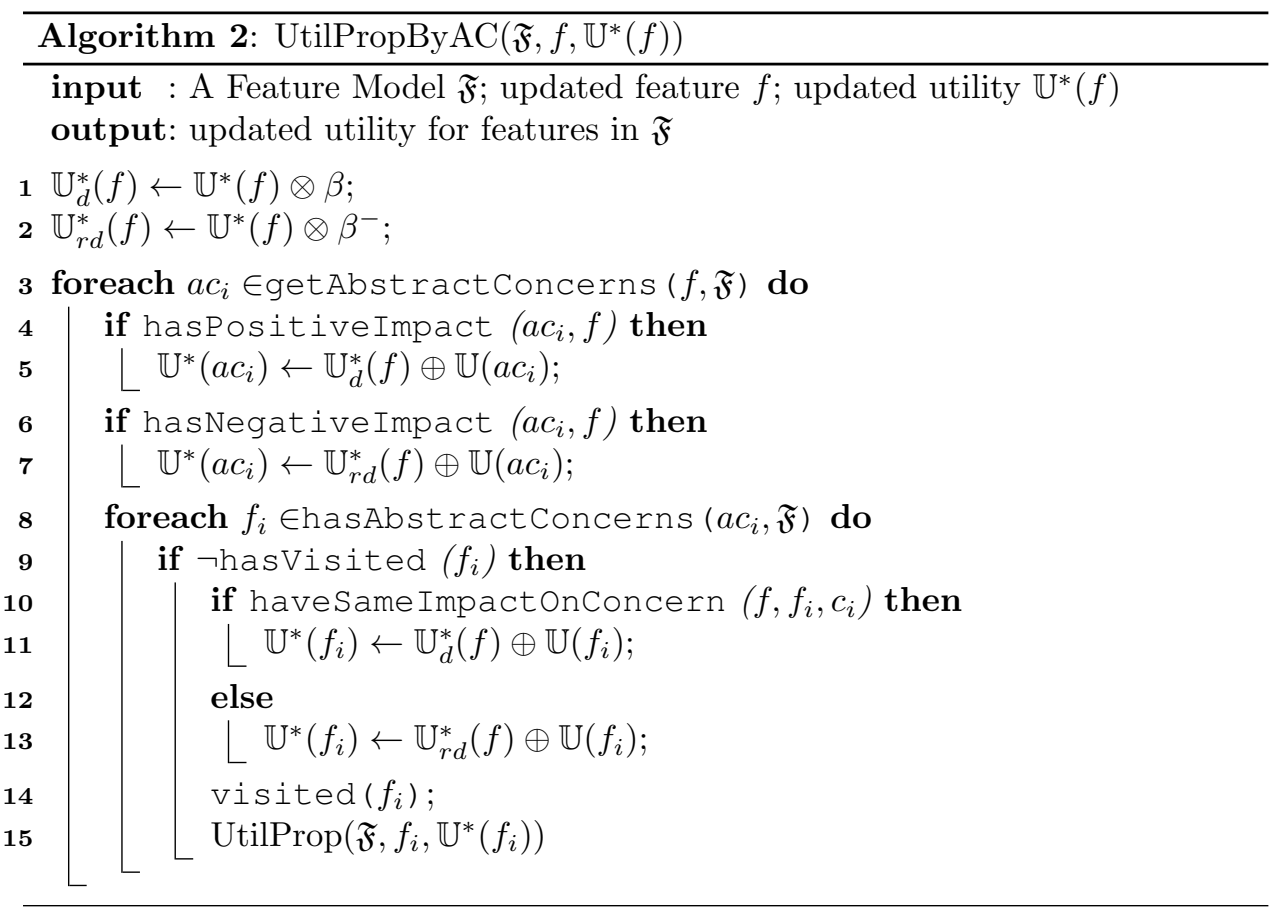

desirable as it also positively impacts abstract concern $a c$. The two forms of utility propagation between features through abstract concerns are depicted in Figure 7. As seen in the figure, if the two features $f$ and $g$ impact a shared abstract concern in the same way, i.e., both have a positive impact or both have a negative impact, then the updated utility of the feature in the gamble such as $f$ can be propagated to the other feature $g$. However, if the impact of the features on the abstract concern are different, i.e., one is positive and the other negative, the updated utility information of $f$ needs to be propagated in such a way that it would have a reverse impact on $g$. These two cases are similar to how utility is propagated for the includes and excludes integrity constraints.

An interesting property of this propagation mechanism is that it enables us to indirectly compute the utility of abstract concerns for the stakeholders. As shown in Figure 4, besides the features that can have utility information assigned to them, abstract concerns can also have utility values. So, once the utility of the features are propagated through an abstract concern, the utility of the abstract concern is also updated and maintained. For instance, if feature $f$ is selected in a gamble and it impacts an abstract concern $a c$ then it is possible to not only propagate utility information to other features through $a c$ but also to $a c$ itself as well. This way we are able to keep track of the utility of the abstract concerns and understand how the stakeholders value them without the need to directly ask for such information from the stakeholders.

Algorithm 2 outlines the process of utility propagation through abstract concerns. Similar to the previous utility propagation mechanism, lines 1 and 2 develop both direct and reverse decayed updated utility values to be used instead of $\mathbb{U}^{*}(f)$. On lines 4-7, the algorithm updates the utility of the abstract concerns that are related to the updated feature $f$. If feature $f$ has a positive impact on an abstract concern, 

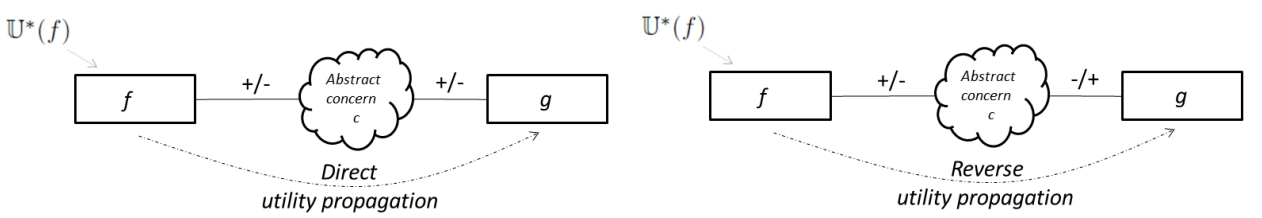

Figure 7. Forms of utility propagation through abstract concerns.

the decayed updated utility of $f$ is propagated to that abstract concern; otherwise, the reverse impact is propagated to the abstract concern. Lines 8-13 propagate the decayed updated utility onto the features related to a shared abstract concern. The propagation of the utility will be direct if both features have a similar impact on the abstract concern and reverse if the features have different impacts on the shared abstract concern. Line 14 ensures that each feature is only impacted once and line 15 will propagate the impact of the update of feature $f_{i}$ onto other features using the propagation through feature interaction mechanism.

The propagation of utility through abstract concern provides the additional benefit of being able to estimate the utility of abstract concerns without having to inquire from the stakeholders and only through observing the interaction of the abstract concerns with features involved in gamble queries. It will also additionally infer partial utility values for features that do not interact directly with each other and are only related through abstract concerns.

Example 3 Lets assume that Figure 8 represents a sub-part of a feature model with one abstract concern ac. Both $f_{1}$ and $f_{3}$ impact ac in a positive way while $f_{2}$ has a negative impact. Now, further suppose that $f_{3}$ has been selected in a gamble as the desirable feature. Based on this, the utility of $f_{3}$ can be updated to $\mathbb{U}^{*}\left(f_{3}\right)$. Given that $f_{3}$ has a positive impact on ac, it is possible to update the utility of ac with a direct decayed utility from $f_{3}$. Similarly given that $f_{1}$ has a positive impact on ac then the updated utility of $f_{3}$ can be propagated to $f_{1}$ as well. However, since the impact of $f_{2}$ on ac (-) is different from the impact of $f_{1}$ on ac $(+)$ then the reverse decayed utility of $f_{1}$ is propagated to $f_{2}$.

\subsection{Gamble Query Formulation and Selection}

It is now important to discuss how gamble queries are dynamically formulated based on the structure of a feature model (Step 1 of Figure 3). As already mentioned, gamble queries present the stakeholders with two competing features and require the stakeholders to select one or the other by both considering the functional desirability of the features and their impact on abstract conerns. It is clear that the source of competition between features in a feature model is the points of variability, which is either the alternative sibling arrangment of the features or the excludes integrity constraints. Therefore, the choice for the competing features in the gamble queries would be features that 1) have an alternative feature group relation with each other; or 2) compete with each other through an excludes integrity constraint. We refer to these two types of competing features as direct competitors. For instance, a gamble query for the feature model shown in Figure 2 would be the choice between BFS or DFS, which are considered direct competitors given their alternative feature group relation. 


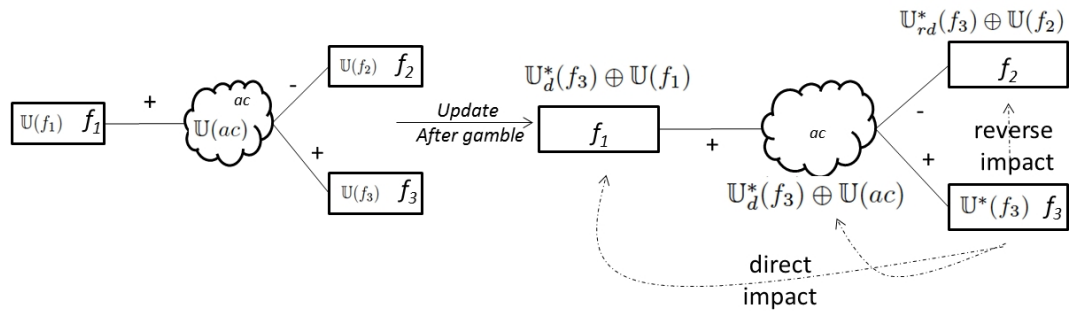

Figure 8. Example utility propagation through abstract concerns.

However, in light of the fact that the outcome of a gamble query in our approach does not only impact the utility of the two features involved in the gamble, but would also benefit from two utility propagation mechanisms that update the utility of related features, we propose the concept of Horizontal Sub-inconsistent Feature Pair (HSFP) in order to extend the two types of competing features that were introduced above. In essence, the idea behind HSFP is that not only do direct competitors have the ability to form suitable gamble queries, but also features that are related to direct competitors could also form gamble queries. As an example, in the above case, BFS and DFS are direct competitors and can form a gamble query. However, we can form other gamble queries based on this competition involving other features. For instance, given that DFS includes cycle detection in the feature model description, we can infer that BFS and cycle detection are also competing features. Hence, we could also formulate a gamble query between these two features. Based on this reasoning, we define HSFP to allow for the formulation of such gamble queries.

Let us first define the horizontal dependency function.

Definition 8 Let $f$ be a feature in the feature model $\mathfrak{F}$, the horizontal dependency function of $f$, denoted $\mathcal{H}(f)$ returns an exact set of features with the following characteristics:

1. $\mathcal{H}(f)$ is minimally defined with at least one member $f$;

2. if $\exists(h$ includes $g) \& h \in \mathcal{H}(f)$ then $g \in \mathcal{H}(f)$;

3. if $\exists(h$ hasAndSibling $g)$ \& $h \in \mathcal{H}(f)$ then $g \in \mathcal{H}(f)$.

The horizontal dependency function develops a set of interdependent features surrounding a feature such as $f$ that are connected to each other either through a sibling and relationship or an includes integrity constraint.

Definition 9 Let $\mathcal{D C}=\left\langle f_{1}, f_{2}\right\rangle$ be a pair of two features which are direct competitors. We define a Horizontal Sub-inconsistent Feature Pair (HSFP) for $\mathcal{D C}$ as $\operatorname{HSFP}(\mathcal{D C})=\left\langle\mathcal{H}\left(f_{1}\right), \mathcal{H}\left(f_{2}\right)\right\rangle$ where $\mathcal{H}$ is the horizontal dependency function.

A horizontal sub-inconsistent feature pair is an extension of the direct competitor concept where the horizontal dependency of each of the direct competitors is employed instead of the features themselves.

Example 4 Let $\mathcal{D C}=\langle D F S, B F S\rangle$ where both DFS and BFS are direct competitors according to Figure 2. $H S F P(\mathcal{D C})=\langle\mathcal{H}(D F S), \mathcal{H}(B F S)\rangle$ where $\mathcal{H}(D F S)=$ $\{D F S, c y c l e$ detection, strongly connected $\}$ and $\mathcal{H}(B F S)=\{B F S\}$. 
Based on Definition 9, it is possible to define more than one gamble query for each pair of direct competitors by using their horizontally dependent features. For instance in the above example, it is possible to formulate three different gamble queries based on the cartesian product of features in $\mathcal{H}(\mathrm{BFS})$ and $\mathcal{H}(\mathrm{DFS})$.

It can be seen that each pair of direct competitors has one corresponding HSFP; therefore, given $n$ direct competitor pairs, we will have $n$ HSFP sets. In order to be able to pose a gamble query to the stakeholders, first we need to decide which HSFP is the ideal set from amongst the $n$ sets and which gamble query from within that HSFP should be posed to the stakholders. We define the concept of expected utility for making such a decision (Step 2 of Figure 3).

Definition 10 The expected utility of a gamble consisting of two features $f_{1}$ and $f_{2}$, denoted $\mathbb{E} \mathbb{U}\left(f_{1}, f_{2}\right)$ is as follows:

$$
\mathbb{E} \mathbb{U}\left(f_{1}, f_{2}\right)=\min _{f_{i} \in\left\{f_{1}, f_{2}\right\}}\left(\sum_{g \in \operatorname{prop}\left(f_{i}\right)} \mid \triangle\left(b_{\left.\mathbb{U}^{*}(g)\right)}|+| \triangle\left(d_{\left.\mathbb{U}^{*}(g)\right)} \mid\right) .\right.\right.
$$

where $\operatorname{prop}\left(f_{i}\right)$ denotes the set of features that are updated as a result of an update to $f_{i}$ and $\triangle$ represents the change in the value of belief or disbelief of the utility of a feature after the update of that feature.

Informally said, the expected utility of a gamble is the amount of changed belief mass that is assigned to the belief or disbelief dimensions of the utility of the features affected by the result of the gamble through the utility update process that will be undertaken by the utility propagation mechanisms. Now given that the choice of the stakeholders between the two options in the gamble query is not known at this stage, the expected utility of a gamble is at most as much as the minimum expected utility of the features in the gamble and hence the minimization function.

The expected utility of a gamble should enable us to select the best gamble query that reveals the most amount of information about the utility of the features for the stakeholders. We define the best gamble query as the one that has the highest (maximum) expected utility from amongst all of the available gamble queries in the $n$ HSFPs (Step 3 of Figure 3). The approach that we have proposed here for selecting the most appropriate gamble query is equivelent to the decision-theoretic minimax approach whose aim is to maximize the minimum gain [26, 27]. We select the gamble query from all the gamble queries that can be generated from the $n$ HSFPs with the highest uncertainty reduction value, i.e., the largest belief mass change from the uncertainty dimension to the belief or disbelief dimensions.

\subsection{Feature Preference Ranking}

We have explained how our approach is able to automatically generate gamble queries, select the most informative gamble query to be posed to the stakeholders and how the results of a gamble query can be employed to update the utility of the related features and abstract concerns. This process involves various decision points which correspond to the gamble queries that are generated and adapted based on answers to prior gamble queries constituting the fomulation of an adaptive decision path. The main outcome of this whole process is the understanding of the stakeholders' preferences and the identification of the utility of the features and abstract concerns for them. As already shown, the utility of each feature or 
abstract concern is represented as a Subjective opinion and through the utility concept shown in the extended feature modeling metamodel.

Given that all features and abstract concerns possess their own utility information, it is possible to use such utility information to develop a ranking to represent the potential preference of the stakeholders regarding the features and abstract concerns and help the stakeholders select features for the final product and build a partial configuration (Steps 6 and 7 of Figure 3). In order to be able to form such a ranking, we employ the Subjective potency metric [23]. This metric shows the significance of a Subjective opinion and can be used in the ranking purposes.

$$
\operatorname{Potency}\left(\mathbb{U}_{f}\right)=\left\{\begin{array}{cc}
0 & \text { if } u_{\mathbb{U}_{f}}=1 \\
\frac{b_{\mathbb{U}_{f}}-d_{\mathbb{U}_{f}}}{1-u_{\mathbb{U}_{f}}} & \text { else }
\end{array}\right.
$$

This potency metric provides us with the means to create a ranking over the features and abstract concerns utilities where the features and abstract concerns with higher potency values are considered to be more valuable for the stakeholders. Based on this quantification, we develop two rankings where the top features and top abstract concerns are rank-ordered based on the potency of their utility and brought to the attention of the stakeholders as potential features to be selected and included in the final product.

\section{Tooling Support}

AUFM Suite is our in-house Eclipse plug-in for feature modeling that has been built on top of FeatureIDE developed at the University of Magdeburg [28]. The tooling support provided for the proposed adaptive staged configuration process is developed within the general architecture of the AUFM Suite. The integration of the various aspects of the staged configuration process has been done through the use of a unified Eclipse perspective incorporating different views, each of which shows relevant information regarding the configuration process to the users.

There are six main parts that can be employed by the users in the developed Eclipse plug-in as numbered and shown in Figure 9. The first part is the feature model editing and manipulation panel that allows the users to develop, edit, and refine their feature model. This part provides the basic feature model design capabilities as inherited from FeatureIDE. In addition, a new coloring scheme has been developed for the features to denote the desirability of each feature for the stakeholders. The coloring scheme is designed in such a way that covers a spectrum of colors from green to red in a way that more desirable features are shown with lighter green colors and less desirable features are shown with darker red. The desirability of the features is calculated based on the potency of the utility of the features obtained through the staged configuration process. As the utility of the features are updated based on the feedback obtained from the gamble queries, the colors of the features are also updated to show how desirable the features are. In this example, the colors show that DFS is desirable for the stakeholders while BFS is not. Features with no utility information are colored by default with purple.

The second part of the perspective is a view that enables the product line engineers or product stakeholders to interrelate each feature with the abstract concerns. The users can show how a feature impacts any of the abstract concerns in a positive or negative way. This view is most suitable to be used in the feature model design process during the domain engineering phase. The figure shows that the brute 


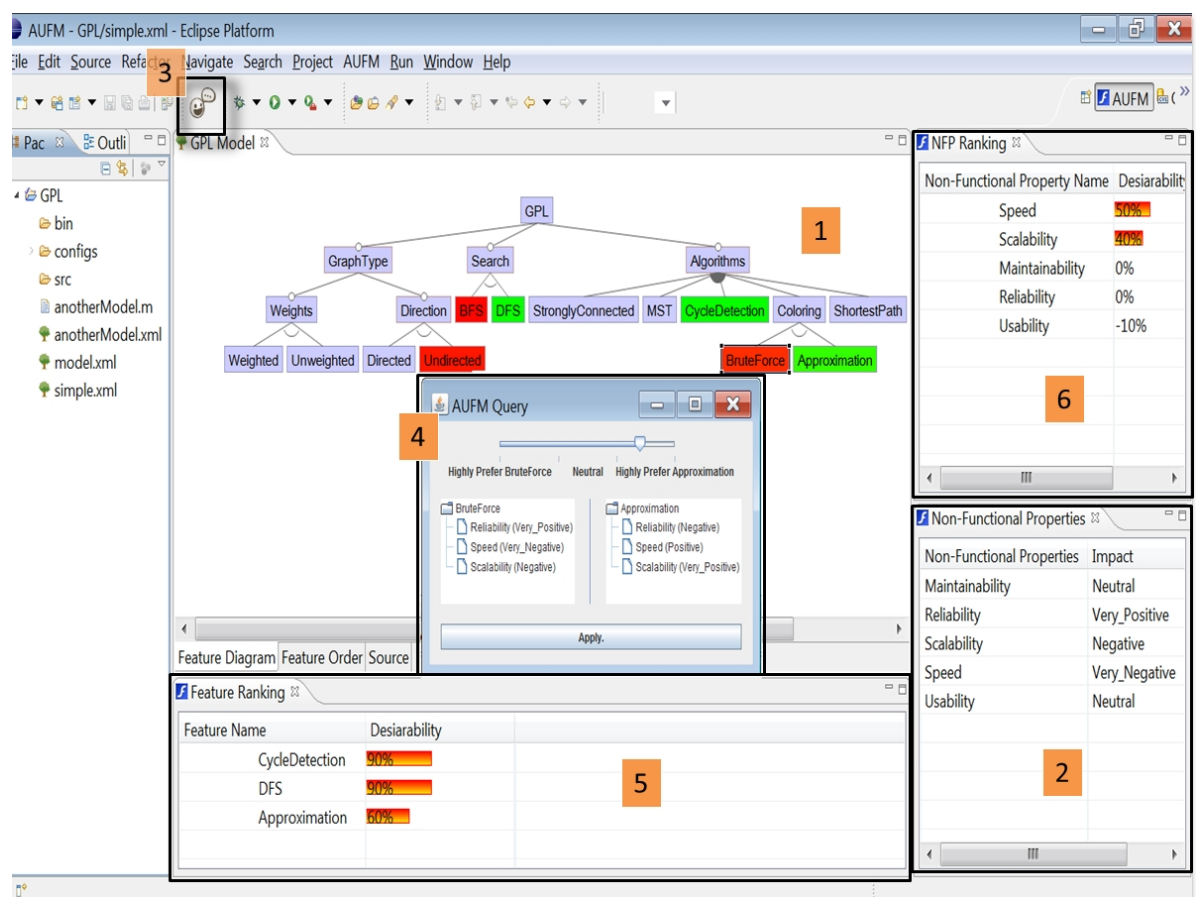

Figure 9. Various parts of the AUFM Suite integrated environment for staged configuration.

force feature impacts reliability in a very positive way while it has a very negative impact on speed.

Once the users are ready to perform the staged configuration process, the integrated tool assists them through the process by asking consecutive gamble queries that will elicit the utility of the features for the users. The users can ask for the next gamble query at any point of time by pressing the gamble query button shown in part three of the figure. A gamble query will be presented to the user where two competing features and their associated abstract concerns are presented. As seen in part four of the figure, the users will have the option to move the slider on top of the dialog to the left or right towards the feature that they more desire. The further the slider is moved to the furthest position on the right or left, the more the users prefer one feature over the other. As soon as a decision is made for the presented gamble, the updated utility information is used to update the utility of other features as well and hence the coloring of the effected features will be updated accordingly.

Besides the coloring of the features that can assist the users in understanding the desirability (utility) of the features, the view in part five of the figure also provides the ranking of the most desirable features. The desirability ranking of the features is performed using the potency measure of the utility of each feature and only features with a positive potency value are descendingly ordered where the most desirable feature is ranked first. In addition, as the utility of features are elicited, the utility of the abstract concerns are also computed indirectly based on the relation of the abstract concerns with features presented in gamble queries. A rank order similar to feature ranking is also presented for the abstract concerns as shown in part six of the figure. With the ranking of abstract concerns, the users will know how their 
decisions regarding features in the gambles affect the abstract concerns. In other words, how will the abstract concerns be impacted as a result of feature selections. In this example, speed and scalability are the two abstract concerns that will be satisfied given the current utility values of the features and usability is the abstract concern that will be negatively impacted.

\section{Exploratory Study Design}

In order to perform an informal evaluation of the developed staged configuration process, we performed two studies. The main objective of these exploratory studies is to informally investigate the suitability of the approach from four aspects. In this section, we introduce these four aspects and describe the environmental setup of the studies.

\subsection{Main Design Drivers}

The approach that we have taken towards the informal evaluation of our work is to investigate the impact of the proposed staged configuration process and its tool support on the performance of a group of users (e.g., application engineers or product stakeholders). We are interested in understanding the effect of our proposed work from four aspects on the users' performance, namely from the rank order, coverage, acceptability and usability perspectives. These four perspectives are defined as follows:

- Rank order shows whether the utility elicitation mechanism incorporated in the staged configuration process is able to correctly reveal the utility of the features for the stakeholders and in doing so rank order the set of desirable features in a way that are in accordance with the preferences of the stakeholders;

- Coverage evaluates how well our proposed approach is able to understand the preferences of the stakeholders and offer recommendations that are in line with the choices that the stakeholders actually have in mind. In other words, assuming that we are aware of the list of desirable features for the stakeholders, coverage would show how well that list is actually reflected by the feature recommendations provided by our approach. This can be seen as similar to the recall measure in information retrieval;

- Acceptability is the measure of recommendation adoption by the stakeholders. This measure shows how many of the recommendations made by our proposed approach are actually being accepted by the stakeholders in their decision making process. This is similar to precision measured in information retrieval;

- Usability refers to the ease of use and learnability of our proposed approach in the context of product configuration in the application engineering phase. In other words, in usability we would like to evaluate whether our approach is able to reduce the complexities associated with the configuration process for the users in such a way that it is desirable for them to use and easy to learn. 
Table 2. The feature models used in the explorations.

\begin{tabular}{|c|c|c|c|c|c|}
\hline Reference & Feature Model & NF (optional, mandatory, grouped) & NVC & DT & CTCR \\
\hline $\mathcal{O}_{1}$ & Billing & $88(45,11,31)$ & $3.87 \mathrm{E}+12$ & 4 & $65 \%$ \\
\hline $\mathcal{O}_{2}$ & Electronic Shopping & $290(82,75,132)$ & $4.52 \mathrm{E}+49$ & 12 & $11 \%$ \\
\hline
\end{tabular}

\subsection{Environment Setup}

7.2.1. Objects of Study The main objects used in our informal studies are feature models that have been obtained from Software Product Line Online Tools (SPLOT) [29]. We have selected two of the largest feature models that are currently available in SPLOT shown in Table 2. The columns of the table represent Number of Features (NF), Number of Valid Configurations (NVC), Depth of model Tree (DT), and Cross-Tree Constraints Ratio (CTCR).

As seen in this table, both of the feature models have a large number of features which would be cumbersome for a user to go through manually. In addition, both models have a high degree of variability where the number of optional and grouped features are quite higher than the number of mandatory features. Also the number of valid configurations that can be derived from the feature models is very high. These characteristics of the two feature models make them ideal to be employed as objects in our studies as they will be naturally hard for the users to process without additional support.

Once these two feature models were selected as the objects of study, we performed an additional processing step on them to annotate the features of the two feature models with abstract concerns. This process was done by the experimenters and benefited from the AUFM tool suite for specifying the relation and degree of impact of each individual feature on the relevant abstract concerns. The abstract concerns consisted of four business concerns and four non-functional properties. The business concerns included time-to-market, development cost, customer satisfaction, and system security. For the non-functional properties, we selected the URPS properties, namely usability, reliability, performance, and supportability [30]. At the end of this annotation process, features were connected with appropriate abstract concerns; and their degree of impact was specified.

7.2.2. Interacting with Subjects The main objective of our informal study was to determine at an early stage whether our proposed staged configuration process and the supporting tool suite is able to assist the application engineers and product stakeholders in deriving a desirable final product. In other words, would the application engineers perform their tasks more efficiently while using the proposed staged configuration process or not. Therefore, our study was performed with the help of human subjects. In our experiments, twelve volunteers actively participated in the evaluations. The participants were all graduate students of Computer Science with the age range between $24-30$ years. The participants all had a good knowledge of software engineering principles and were given a mini-tutorial on aspects of software product lines and feature models prior to the study period. Different phases of the product line engineering lifecycle including the phases of the application en- 
gineering process and details of staged configuration were also explicitly explained and the aim and artifacts of this phase were detailed for them.

In order to form a fair distribution of activities between the two objects of study, the subjects were randomly split into two groups. Each of the subjects in both groups were asked to individually take part in two separate experiments. The first group was asked to employ $\mathcal{O}_{1}$ first for the first experiment and then the subjects were later asked to use $\mathcal{O}_{2}$ to perform the second experiment. Both experiments were performed using the proposed staged configuration process and its supporting tool. The second group of subjects were asked to perform the same procedure but by using $\mathcal{O}_{2}$ for the first experiment and $\mathcal{O}_{1}$ for the second experiment. One experimenter was always present during both of the experimenting periods. The experimenter employed a shadowing method to only observe the performance of the subject during the experimentation periods. This experimental setup was used to minimize the effect of study objects and experimenters on the performance of the subjects and hence support the validity of the obtained results.

\section{Preliminary Observations}

The goal of our informal studies was to validate whether the proposed staged configuration process with feature utility elicitation and dynamic decision points has any positive impact on the performance of the users in terms of rank order, coverage, acceptability and usability. For this purpose, we report and analyze our early observations with regards to these four aspects.

\subsection{Rank-ordering of Recommendations}

The main purpose of our proposed staged configuration process is to identify the utility of the features of a feature model for the stakeholders. It is clear that different stakeholders have different preferences and hence based on the stakeholder performing the staged configuration process, the utility of the features would be different. On the other hand, research has already shown that human subjects are not comfortable with directly providing and assigning accurate utility information to available options [31]. Therefore, in the first experiment, we decided to engage with the study participants in a way that allows them to comfortably work with the proposed staged configuration process and also provide feedback on its performance. In order to achieve this, each of the participants was asked to load the feature model that she was assigned for experiment one $\left(\mathcal{O}_{1}\right.$ for group 1 and $\mathcal{O}_{2}$ for group 2) into AUFM suite. They were then asked to familiarize themselves with how the features impacted the abstract concerns by going through the features of the feature model as they desired and by looking at the related abstract concerns and the impacts.

After the familiarization step, the participants interacted with the feature model by requesting gamble queries. As mentioned earlier, each gamble query represents a decision point in our model and the sequence of the decision points form a decision path. The participants were asked to constantly interact with the tool through gamble queries in order to understand their own preferences. Once the participants felt comfortable with the feature model and believed that they had a good understanding of the features, each of them was asked individually to select the top ten features that she thought were most desirable. Each list would then be ordered descendingly based on the order of preference by that participant. The list prepared 
Table 3. The correlation between explicit and predicted feature ranks.

\begin{tabular}{lll}
\hline Participants & Kendall's $\tau$ & p-value \\
\hline $\mathcal{P}_{1}$ & $\mathbf{O . 5 7}$ & $\underline{\mathbf{O . 0 6}}$ \\
$\mathcal{P}_{2}$ & 0.77 & 0.002 \\
$\mathcal{P}_{3}$ & $\boldsymbol{O . 5}$ & $\underline{\mathbf{O . 1}}$ \\
$\mathcal{P}_{4}$ & 0.64 & 0.03 \\
$\mathcal{P}_{5}$ & 0.86 & 0.0006 \\
$\mathcal{P}_{6}$ & 0.90 & 0.006 \\
$\mathcal{P}_{7}$ & 0.82 & 0.001 \\
$\mathcal{P}_{8}$ & 0.95 & 0.0001 \\
$\mathcal{P}_{9}$ & $\boldsymbol{O . 5}$ & $\underline{\mathbf{O . 1}}$ \\
$\mathcal{P}_{10}$ & 0.78 & 0.009 \\
$\mathcal{P}_{11}$ & 0.92 & 0.001 \\
$\mathcal{P}_{12}$ & 0.64 & 0.03 \\
\hline Overall & 0.74 & 0.038 \\
\hline
\end{tabular}

by each participant was then compared with the list proposed by our tool in the form of feature rankings (part 5 of Figure 9). The idea behind the comparison is that the more these two lists are similar to each other, the better the tool has been able to identify and model the utility of the features for that participant.

In order to compute the similarity between the two lists, i.e. the list preferred by a participant and the list proposed by our tool for that participant, we employed Kendall's rank correlation coefficient $(\tau)$. The Kendall $\tau$ test is a non-parametric hypothesis test that measures rank correlation. In other words, it computes the similarity of the orderings of a set of data points when generated by different processes. We also calculated the p-value for this significance test, which shows the role of coincidence in the observed correlation. A p-value of less than 0.05 is often considered to be a sign for a statistically significant correlation observation. The results of our observation of Kendall's $\tau$ test are shown in Table 3 .

As seen in the Table, we observed significant correlation $(p$-value $<0.05)$ between the feature rankings on the participants' list and that on the recommended list by the tool, which is the result of the utility elicitation process. From the twelve participants in our studies, there were only three participants, namely $\mathcal{P}_{1}, \mathcal{P}_{3}$ and $\mathcal{P}_{9}$ whose preferences did not significantly correlate with the feature recommendations. This means that the recommendations offered based on the utility elicitation process were not accurate for these three participants. On the other hand, a significant correlation was observed for the other nine participants that shows that the ranking of features proposed for each individual stakeholder was highly resemblant of the feature preferences for the stakeholders in $75 \%$ of the cases of this preliminary study. Overall, we observed that in $75 \%$ of the subjects, the utility elicitation process has been able to offer recommendations that are significantly correlated $(0.038<0.05)$ with the list of desirable features for each individual participant with an average $\tau=0.74$.

Based on the observations of this first preliminary experiment, we believe initial favorable indication for the proposed utility elicitation process exists which shows that it would be successful in predicting the utility of the features for the stakeholders and providing a list of recommended features that are close to the preferences of the stakeholders. 

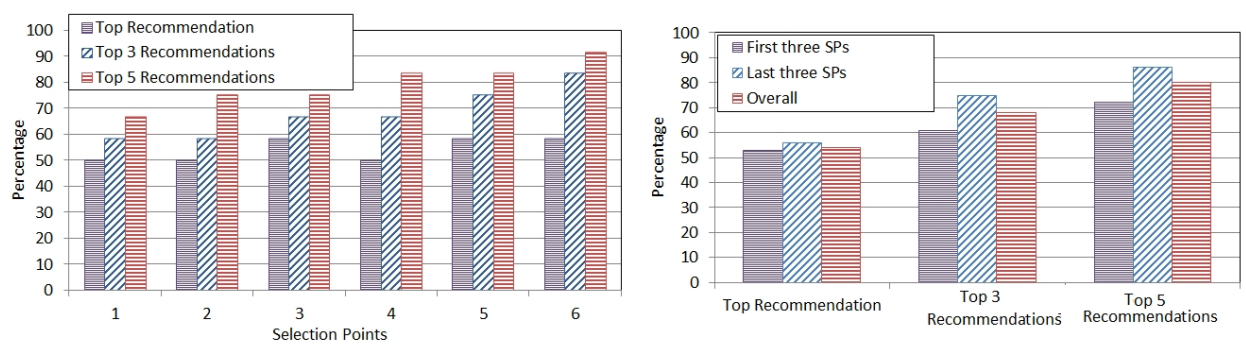

Figure 10. The coverage at each selection point.

\subsection{Recommendation Coverage and Acceptability}

In the second study, the participants were asked to use the other feature model for the experiment $\left(\mathcal{O}_{2}\right.$ for group 1 and $\mathcal{O}_{1}$ for group 2). Similar to the first study, the participants were asked to load the feature model into the AUFM Suite and explore the interaction between the features and the eight abstract concerns used to annotate the features. However, unlike the first study where the participants interacted with the tool through the gamble queries and only provided a ranked list of ten features when they felt comfortable with the feature model, in this second example, the stakeholders were asked to make six feature selections. In order to make the six selections, the participants were asked to make gradual progress in the form that they would first interact with the tool through several gamble queries then make a selection decision and then repeat this process until the six decisions were made.

We refer to the point in time when a participant makes a feature selection as a selection point. Given that the most important action of a participant at each selection point is to identify the most suitable feature to be included in the final product, we observed how well the feature recommendation model, which is based on the elicited utility for the participant, was able to identify the most suitable feature to be selected for each case. In other words, we are interested in finding out about the coverage of our proposed work by observing whether the feature actually selected by the participant at a selection point is present in the list of recommended features by our tool. However, given that the list of recommended features includes a rank-order of all features with a positive utility, we restrict the coverage computation to the top, top three and top five features, i.e., whether the feature selected by the participant is the same as the top feature, is among the top three or is among the top five features in the recommended list provided by our tool.

The observed coverage of our approach for this limited experiment is shown in Figure 10. As shown in the figure on the left side, the coverage of the recommendations provided in each selection decision in all three forms (top, top three and top five) increases from one selection point to the next. The main reason behind this is that in order for a participant to be ready to make a decision at a selection point, she would need to complete several decision points during which gamble queries will be presented to the participant. The answers to these gamble queries will increase the knowledge about the utility of the features and will hence improve the accuracy of the recommendations. In this informal exploration, the probability that the feature selected by a participant at the first selection point be exactly the one on top 


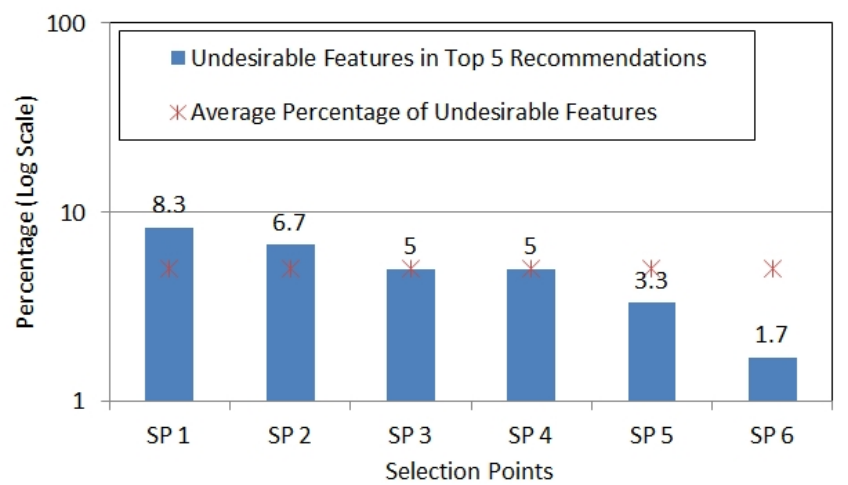

Figure 11. The percentage of undesirable features in the top five list.

of the recommendation list is on average around $50 \%$ while this increases to close to $60 \%$ in the sixth selection point. Similarly, the probability that the selected feature is among the top five recommended features is around $65 \%$ in first selection point whereas it is much higher in the sixth round with over $90 \%$. These are positive indications of the potential of our work in that it shows that even in the worst case which happens in the first selection point, the feature selected by the participant is expected to be observed in the top five recommended features by our tool in $65 \%$ of the cases.

The right hand-side of Figure 10 summarizes these observations from a different point of view. Based on this view, the feature selected by a participant in any of the six selection points is likely to be exactly the same as the top recommended feature by our tool in over $54 \%$ of the time. Furthermore, the selected feature is amongst the top three and top five features with the probability of $68 \%$ and $80 \%$, respectively. This shows that the participants in our experiments were able to greatly benefit from the feature recommendations (especially by looking at the top five features) as there is at least an $80 \%$ likelihood that their most desirable feature is one of the features in this list.

Now, in order to further observe the coverage of our recommendation model, the participants were also asked to look at the top five features recommended by the tool and mention how many of these five features they believe should not have been included in the top five list. In other words, how many of the features they thought were falsely classified as one of the top five. Figure 11 shows the percentage of undesirable features in the top five list as specified by the participants. Similar to Figure 10, the recommendation list becomes more accurate as the participants move along in the selection points. It is noticeable that the percentage of falsely classified features in the top five recommended features is in the worst case $8.3 \%$ in the first selection point and even drops further to $1.7 \%$ in the sixth selection point. On average, there is the low $5 \%$ probability for a completely undesirable feature to be mistakenly classified as one of the top five features.

With regards to acceptability of the feature ranking provided by our tool, the participants were asked to provide feedback on whether they would have accepted the suggestions of the tool at each selection point. The idea behind acceptability was not only to see whether the utility of the features for participants have 


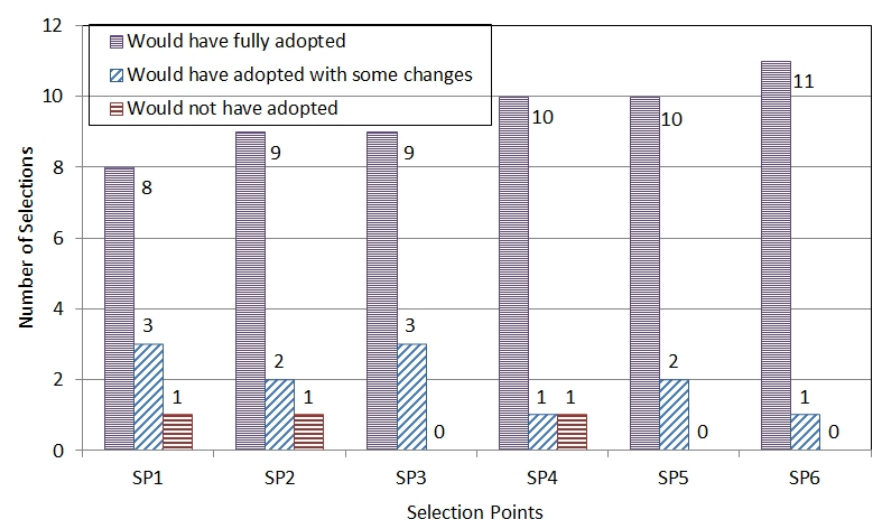

Figure 12. The acceptability of recommendations at each selection point.

been identified properly and that the feature ranking is reasonable, but also to observe whether the participants adopted the recommendations in practice as well. To evaluate this, the participants were asked to select one of the following three options at each selection point: i) I am comfortable with adopting the top five recommendations provided by the tool at this selection point; $i i$ ) I feel comfortable with accepting the top five recommendations of the tool after some re-ordering of the features; iii) I do not feel comfortable adopting the recommendation. Figure 12 shows the responses received from the participants regarding acceptability.

As seen in the figure, in all six selection points at least $91 \%$ of the participants selected either the first or the second option showing that the recommendations provided by the system is at least acceptable with some minor re-arrangement of the order of the features. From the $91 \%$ who selected either the first or second options, the number of participants that selected the first option who were comfortable with fully adopting the recommended features grew steadily from $67 \%$ to $91 \%$ from the first selection point to the sixth selection point. This is a significant observation in that it shows that as the participants interact more with the tool, the utility elicitation process is able to more accurately model their preferences and provide more precise and acceptable recommendations. Furthermore, even in the worst case which happened in the first selection point, only one participant (9\% of participants) was uncomfortable with the proposed recommendations. The rest of the participants felt comfortable in accepting and trusting the feature recommendations offered by the tool based on the derived feature utilities.

According to the observations made from the feedback obtained from the study participants, we believe that the proposed staged configuration process has the potential to effectively model feature utilities for the stakeholders and offer acceptable and covering feature recommendations based on the utilities.

\subsection{Usability of Approach}

There is always the possibility in the area of software engineering that performance does not necessarily entail usability. In other words, techniques that yield better performance and more accurate results are not necessarily adopted and used by the community because of difficulty in employing the tools or technologies. For 
this reason, given that the underlying formalism for the proposed feature utility elicitation process is rather complex, we wanted to make see whether this complexity is imposed on the application engineers or product stakeholders in the staged configuration process or not. To this end, the participants were asked to complete a five-question questionnaire. The questionnaire asked the participants to provide their feedback regarding each of these five questions using the five-point likert scale, i.e., strongly agree, agree, neutral, disagree and strongly disagree. The five questions that were asked from the participants are as follows:

1. (Eases the configuration process): The proposed staged configuration process not only does not add any additional difficulty to the development of a product configuration, but also eases the decision making process and makes the completion of the process easier.

2. (Enhances desirability of end result): The products that are developed based on the proposed staged configuration process have a higher likelihood of been more desirable for the stakeholders because a more systematic approach is used for understanding the preferences of the stakeholders.

3. (Reduces cognitive complexity): When exploring the feasible feature space of a software product line, given the large size of the feature model, the amount of information related to the feature model could pose a heavy mental burden on the application engineers or the product stakeholders. The proposed approach is able to reduce the mental burden to a more manageable level.

4. (Provides insight into feature interaction): The interaction with gamble queries and the update of feature utility information through the coloring scheme in the presented tool is able to provide more insight into feature interactions which would be otherwise hard to understand.

5. (Reduces time associated with configuration): The time taken to complete the staged configuration process and developing a final product using the proposed approach could be considered to be significantly less than the time taken to configure a product line without it.

Figure 13 shows the results of the questionnaire. As it can be seen in this figure, the responses have been favorable (either strong agree or agree) for the first three questions. This means that the participants believe that the proposed approach is able to ease the configuration approach, enhance the quality of the final product and also reduce the cognitive complexity associated with the configuration process. Regarding the fourth question, ten of the twelve participants believe that the proposed approach is able to reveal more information regarding feature interactions and there are only two participants who are not sure whether it makes any impact or not. In the last question, there are half of the participants who are neutral regarding the effect of the proposed approach on the reduction of time spent on configuration. However, still the other half of participants agree that this could reduce the time for configuration. It seems that the time spent in the configuration process is a measure of the rigor of the participants and hence we believe that it should naturally not reduce with the employment of the proposed staged configuration process.

Overall, there have been no negative reports in the usability questionnaire for any of the five questions by our participants. Based on the informal feedback from the 


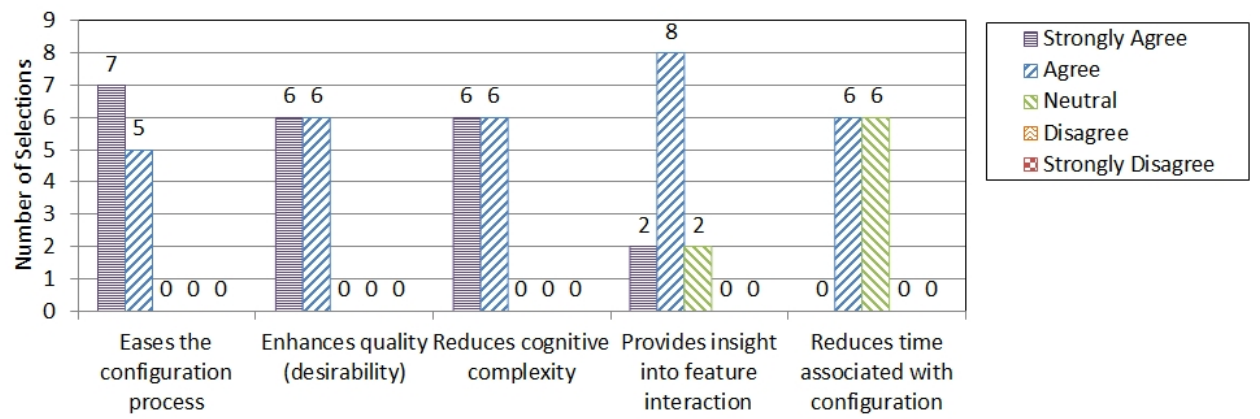

Figure 13. The result of the usability questionnaire.

participants in the form of the questionnaire, this could potentially show that the participants were interested in adopting this approach for the configuration process and hence we believe that further customization and enhancement of the tool could make it quite usable for the application engineering process.

\section{Discussions}

\subsection{Lessons Learned}

The process of the design and execution of the experiments required for this research work provided us with some insight into the role of support systems in the area of software product lines and especially for the application engineering phase of the lifecycle. It should be noted that the lessons learnt were the side product of the informal studies and have not been rigorously validated through further experiments; nevertheless, we believe they are interesting observations.

One of the main issues that draw our attention was the influence of the utility elicitation process on the time taken to perform and complete a staged configuration process. Despite our best hopes, it turned out that half of the participants believed that the provided tool suite and the technology behind it did not have an impact on reducing the time associated with staged configuration. This was an interesting observation for us given our previous experience. In a related work, we developed a decision support platform for the domain engineering phase of the software product line lifecycle [6]. In our experiments and through the actual tracking of the time taken to perform a domain engineering process with and without the decision support platform, we observed that the time was quite similar for both settings. Although in our experiments in this current paper, we did not measure the time taken to perform the tasks, due to a lack of comparison benchmark, the opinions expressed by the participants regarding the impact of the tool on time taken to complete the task resembles our previous observation that no significant impact can be observed. We think that it is possible to conclude that the use of decision support platforms, such as the one developed in this current paper and also our earlier work for the domain engineering phase [6], does not necessarily reduce the time associated with performing the task but it does certainly impact and improve the quality of the artifacts that are developed as a result.

The second point that was observed during feature model configuration was the emphasis that the participants had on understanding the impact that their deci- 
sions had on abstract concerns. The observations by the experimenters showed that the participants were interested in being cognisant of the state of the abstract concerns by frequently checking their ranking after answering gamble queries and also answering gamble queries by considering the associated abstract concerns. In our opinion, this is an important observation as the role of non-functional properties and business concerns have not been central in the product line configuration literature. Functional aspects that conveniently map to features in the feature model have received more focus while it appears from our observations that the stakeholders or application engineers are at least equally interested in the effect of abstract concerns on the final product. This is an indication that configuration methods that allow for both functional and non-functional optimization in the configuration process could be of interest to the users.

The other issue that was encountered during the experiments was the desire of some participants to override the results of the utility elicitation process. This specifically happened a couple of times for some participants who did not agree with some of the information that was elicited and was reflected through the coloring of the features. The participants thought that some of the features that were colored should have a different color or at least should have a different lighter or darker shading. For instance, one feature was colored with light green and the participant believed that given that she is sure about the feature being undesirable that it should be colored dark red. In such cases, the participants liked to have the opportunity to override the derived utility information with their own belief. This was a functionality that was not present in our tool. Our conclusion was that the participants should have the capability to correct the support system when they feel that it has been misled but at the same time we believe that not too much control over the support system should be given to the user due to the point that the support system might be improving itself through gradual observation but an untimely intervention from the user might disrupt a plan that is on the right track.

\subsection{Limitations}

We would like to highlight what could be seen as the limitations of our approach. The first restriction of our approach is that it is designed to work most effectively within the software product line domain where a feature model representing the finite set of features of that domain already exists. This is a requirement for our work that allows us to identify the set of competing features and build gamble queries. It is possible to extend our work to support a general requirements prioritization process but the current work does not extend into that area. The other requirement of our approach is that we assume that the application engineers and/or the available stakeholders are capable of understanding the goals of their target system and are able to translate those goals into priority information that would allow them to decide between two competing features in the gamble queries. There might be situations when the stakeholders cannot decide between two competing features in which case our approach would not provide them with any additional support. In such circumstances, the stakeholders can benefit from other decision making tools such as our S-AHP technique [32] to identify priority and decide on their option in the gamble query.

It is also important to point our that the empirical studies reported in this paper are preliminary exploratory studies that intend to present some initial observation on the potential of our approach. It should be noted that further controlled ex- 
perimentation is required to determine the real impact of this approach under real world circumstances. Some of the limitations of our exploratory study that we can point to are the following: In our study, a limited number of data points were collected due to our restricted access to appropriate participants that had enough knowledge of software engineering within the area of software product lines. In addition, we only worked with two publicly available feature models from the SPLOT repository, which were the largest in the collection and hence were most suitable for our experiments. Although these two feature models were the largest that we had access to and in light of the fact that using smaller feature models would not have in any way presented us with better and more generalizable results, this limitation may pose threats to the drawn conclusions. For instance, the use of a larger number of feature models could have impacted the accuracy of our conclusions.

The other limitation is a result of using subjective measurement mechanisms for measuring the opinion of the different participants through the use of the likert scale. Participants may have different attitudes towards the evaluation of the dependent variables. For instance, some participants may be reluctant to provide high subjective values to the options that they are evaluating, whereas the others may have quite a different mind-set, which could lead to a validity threat. We need to recruit more number of participants in order to smooth out the effect of this issue. Another threat to the studies could be the presence of one experimenter in all experiments alongside the participant. Although the experimenter acted through a shadowing method, still the pressure from mere presence of the experimenter could have impacted the performance of the participants. We are now considering the use of virtual presence for the experimenter, such as video-conferencing, to reduce the impact.

\section{Related Work}

Most efforts in feature-oriented representations of product lines has been on the development of automated algorithms for product line configuration. More specifically most of these techniques have been formulated to address the automated configuration of software product line feature models based on stakeholders' functional and non-functional requirements $[33,34,35,36,37]$. Feature model configurations have been often derived, validated and verified using Logic Truth Maintenance Systems (LTMS). Three of the most widely used methods in this context are Constraint Satisfaction Problem (CSP) solvers [38], propositional SATisfiability problem (SAT) solvers [35, 39] and Binary Decision Diagrams (BDD) [40]. Furthermore, Description Logic reasoners have also been employed to validate the structural correctness of feature model configurations [41, 36]. The main goal of these approaches is to take as input a set of functional and non-functional requirements from the stakeholders and then develop a configuration that maximally satisfies these requirements. However, as Czarnecki et al have pointed out, the configuration process should be performed in a step-by-step manner through which consecutive specializations are formed in order to build the final configuration [5]. Therefore, although the automated configuration methods can be used for specialization, they do not offer methodological support for deriving and understanding the requirements of the stakeholders.

Some researchers have proposed the idea of prioritizing features during the design phase (domain engineering phase) in a way that would facilitate feature selection during product derivation phase [22]. The idea of prioritization of features is inter- 
esting, but it does not seem to be feasible given that feature priorities change based on the target stakeholders and the context where the product line is being specialized and configured; therefore, priority values for features should be developed during the specialization process. To address this, we have previously developed the Stratified Analytic Hierarchy Process (S-AHP) that allows for the prioritization of software features based on stakeholder feedback [32]. The process is a restricted form of AHP for software product lines in that it reduces the number of required pairwise comparisons by considering abstract concerns. However, it is possible that this approach faces a high number of pairwise comparisons if the number of abstract concerns or comparable features grow.

Closely related to understanding the utility of features, the requirement engineering community has significantly contributed to the development of requirement prioritization techniques for requirements selection [42, 43]. In this context, one suggestion for prioritizing requirements has been the use of requirement priority groups [44] as a way to nest similar requirements together and create an internal rank for requirements in each group. This approach is not so suitable for feature models, since the structure of the feature model tree is of high importance while the decision about the priority and specialization of the feature model is being made; therefore, creating priority groups that would become feature tree structure oblivious might result in irrelevant feature model configurations. Other models such as the use of bubble sort over requirements, cumulative and hierarchical voting have been proposed that are not applicable to feature models given the relative large size of the feature models in software product lines [45].

On the other hand, decision-oriented models inherently allow for a more close integration of stakeholders' opinions compared to feature-oriented models given that the central theme of decision models is decision making that requires the involvement of a decision maker [46]. There have been several foundational work on decision models including DOPLER [47], Synthesis [48] and KobrA [9]. Although slightly different in their formalization, the underlying principle behind the decision models is that decisions can both allow for the representation of variability and also most effectively and unambiguously guide the product customization/derivation process. Typically decisions, assets or artifacts, and dependencies between them are modeled in a decision model in a way that answering a question can lead to providing the value for one or more decisions in the model that advances the product derivation process $[49,50]$. The main deficiency for this type of models is their inflexibility and high maintenance costs. Unlike feature models which are processagnostic, decision models rely on the decision process that is defined in the domain engineering lifecycle. Hence, the need for a small change in the decision process can lead to violation of many dependencies and the need for rearranging the decision making order. Therefore, while decision models are very effective for application engineering, they can be costly in terms of maintenance and update.

In light of the aforementioned significant achievements in the area of product configuration in software product lines and based on the limitations of both featureoriented and decision-oriented models, we have integrated a utility elicitation process into the staged configuration process in a way that it benefits from the structural characteristics of a feature models to dynamically generate decision models that can be used for feature selection and product configuration. Our approach complements feature-oriented models by helping to elicit the stakeholders requirements and identify their preferences over the available features and also improves 
decision-oriented models by building the decision models dynamically; hence, reducing the costs associated with maintenance and update of the models.

\section{Concluding Remarks}

The product line engineering paradigm has been recognized as one of the effective means for software reuse and rapid software development. This paradigm benefits from a development lifecycle that has two distinct but interrelated phases, namely domain engineering and application engineering. The focus of the work presented in this paper is to facilitate the task of the application engineers or product stakeholders in the application engineering phase by helping them understand the utility of the features available in the product line and identifying their preferences. The utility elicitation process is carefully integrated with an interactive staged configuration process in a non-obtrusive manner allowing the engineers to get engaged with the developed tools without having to be aware of the details of the underlying utility elicitation process. The interaction with the process is through easy-to-understand gamble queries that gradually expose the requirements of the product stakeholders.

Our exploratory empirical evaluations have shown that feature utility elicitation integrated into staged configuration is able to enhance performance during the configuration process from three distinct perspectives: rank-order, coverage and acceptability. This means that the employment of the support tool incorporating the utility elicitation process is able to positively influence the quality of the application engineering phase outcomes. It is noteworthy to mention that the participants in the experiments were quite satisfied with the usability of our tool by mentioning that it eased the configuration process, enhanced the quality of the final product, and reduced the mental burden and cognitive complexity associated with the configuration process. However, there were mixed opinions regarding the impact of our approach in reducing the time needed to build a product configuration. This is in line with our previous finding regarding a decision support platform for the domain engineering phase [6].

We believe that our work provides the following benefits for the application engineering phase: (1) Product stakeholders often have a very high-level view of their needs and it is hard for them to translate that into concrete feature requests and also it is difficult for them to understand the feature structure of a product line. Our work provides a support mechanism that helps the application engineers to elicit the preferences of the stakeholders through gamble queries that ask the stakeholders to answer simple feature comparison questions based on functional properties and abstract concerns; (2) Decision models are more intuitive than feature models for being used in the product derivation process; however, they are less flexible and harder to develop during the domain engineering phase. Our work provides the means to bridge the gap between feature models that have already been developed during domain engineering and dynamically builds a decision model. In the proposed approach, each gamble query is a decision point and the sequence of gamble queries forms a decision path. The advantage of this approach is that the decision path is not predetermined and will be unique to the stakeholder involved in answering the gamble queries; (3) The integrated tool that we have developed on top of the FeatureIDE plugin provides a unique perspective with several views regarding the preferences of the stakeholders. It offers a coloring scheme to show the desirability of each individual feature along with other views showing the ranking of the features and abstract concerns based on their utility. 


\section{References}

1. Czarnecki, K., Eisenecker, U.: Generative programming. Springer (2000)

2. Pohl, K., Böckle, G., Van Der Linden, F.: Software Product Line Engineering: Foundations, Principles, and Techniques. Springer (2005)

3. Weiss, D.M., Clements, P.C., Kang, K., Krueger, C.: Software product line hall of fame. In: SPLC '06: Proceedings of the 10th International on Software Product Line Conference, Washington, DC, USA, IEEE Computer Society (2006) 237

4. Matinlassi, M.: Comparison of software product line architecture design methods: Copa, fast, form, kobra and qada. In: Proceedings of the 26th International Conference on Software Engineering, IEEE Computer Society (2004) 127-136

5. Czarnecki, K., Helsen, S., Eisenecker, U.W.: Staged configuration using feature models. In: SPLC. (2004) 266-283

6. Bagheri, E., Ensan, F., Gasevic, D.: Decision support for the software product line domain engineering lifecycle. Autom. Softw. Eng. 19 (2012) 335-377

7. Dumitru, H., Gibiec, M., Hariri, N., Cleland-Huang, J., Mobasher, B., Castro-Herrera, C., Mirakhorli, M.: On-demand feature recommendations derived from mining public product descriptions. In: Proceedings of the 33rd International Conference on Software Engineering. ICSE '11, New York, NY, USA, ACM (2011) 181-190

8. Bagheri, E., Gasevic, D.: Assessing the maintainability of software product line feature models using structural metrics. Software Quality Journal 19 (2011) 579-612

9. Atkinson, C., Bayer, J., Muthig, D.: Component-based product line development: the kobra approach. In: Proceedings of the first conference on Software product lines : experience and research directions: experience and research directions, Norwell, MA, USA, Kluwer Academic Publishers (2000) 289-309

10. Kang, K., Cohen, S., Hess, J., Novak, W., Peterson, A., INST, C.M.U.P.P.S.E.: Featureoriented domain analysis (FODA) feasibility study. Carnegie Mellon University, Software Engineering Institute (1990)

11. Pu, P., Faltings, B., Torrens, M.: User-involved preference elicitation. In: IJCAI Workshop on Configuration. (2003) 56-63

12. Chajewska, U., Koller, D., Parr, R.: Making rational decisions using adaptive utility elicitation. In: Proceedings of the Seventeenth National Conference on Artificial Intelligence and Twelfth Conference on Innovative Applications of Artificial Intelligence, AAAI Press (2000) 363-369

13. Lee, K., Kang, K.C., Lee, J.: Concepts and guidelines of feature modeling for product line software engineering. In: Proceedings of the 7th International Conference on Software Reuse: Methods, Techniques, and Tools. ICSR-7, London, UK, UK, Springer-Verlag (2002) 62-77

14. Tessier, P., Gérard, S., Terrier, F., Geib, J.M.: Using variation propagation for model-driven management of a system family. In: Proceedings of the 9th international conference on Software Product Lines. SPLC'05, Berlin, Heidelberg, Springer-Verlag (2005) 222-233

15. Lopez-Herrejon, R.E., Batory, D.S.: A standard problem for evaluating product-line methodologies. In: Proceedings of the Third International Conference on Generative and Component-Based Software Engineering. GCSE '01, London, UK, UK, Springer-Verlag (2001) 10-24

16. Kulasekere, E.C., Premaratne, K., Dewasurendra, D.A., Shyu, Bauer, P.H.: Conditioning and updating evidence. International Journal of Approximate Reasoning 36 (2004) 75-108

17. Stephanou, H.E., Lu, S.Y.: Measuring consensus effectiveness by a generalized entropy criterion. IEEE Trans. Pattern Anal. Mach. Intell. 10 (1988) 544-554

18. Pope, S., Josang, A.: Analysis of competing hypotheses using subjective logic. In: 10th CCRTS: The Future of Command and Control. (2005) 1-30

19. Sentz, K., Ferson, S.: Combination of evidence in dempster-shafer theory. Technical report, Sandia National Laboratories, SAND 2002-0835 (2002)

20. Josang, A.: A logic for uncertain probabilities. Int. J. Uncertain. Fuzziness Knowl.-Based Syst. 9 (2001) 279-311

21. Josang, A., Daniel, M., Vannoorenberghe, P.: Strategies for combining conflicting dogmatic beliefs. In: Proceedings of the Sixth International Conference of Information Fusion. (2003) $1133-1140$

22. Czarnecki, K., Helsen, S., Eisenecker, U.W.: Formalizing cardinality-based feature models and their specialization. Software Process: Improvement and Practice 10 (2005) 7-29

23. Bagheri, E., Ghorbani, A.: A belief-theoretic framework for the collaborative development and integration of para-consistent conceptual models. Journal of Systems and Software 82 (2009) 707-729 
24. Bagheri, E., Ghorbani, A.: The analysis and management of non-canonical requirement specifications through a belief integration game. Knowledge and Information Systems 22 (2010) 27-64

25. Bagheri, E., Ghorbani, A.A.: On the collaborative development of para-consistent conceptual models. In: Proceedings of the Seventh International Conference on Quality Software. QSIC '07, Washington, DC, USA, IEEE Computer Society (2007) 336-341

26. Peterson, M.: An introduction to decision theory. Cambridge Books (2009)

27. Fishburn, P.: The foundations of expected utility. Theory \& Decision Library (1982)

28. Kastner, C., Thum, T., Saake, G., Feigenspan, J., Leich, T., Wielgorz, F., Apel, S.: Featureide: A tool framework for feature-oriented software development. In: Proceedings of the 31st International Conference on Software Engineering. ICSE '09, Washington, DC, USA, IEEE Computer Society (2009) 611-614

29. Mendonca, M., Branco, M., Cowan, D.: S.p.l.o.t.: software product lines online tools. In: OOPSLA '09: Proceeding of the 24th ACM SIGPLAN conference companion on Object oriented programming systems languages and applications, New York, NY, USA, ACM (2009) 761-762

30. Pollard, C., Gupta, D., Satzinger, J.: Teaching systems development: A compelling case for integrating the sdlc with the itsm lifecycle. Information Systems Management 27 (2010) 113-122

31. Pu, P., Chen, L.: User-involved preference elicitation for product search and recommender systems. AI Magazine 29 (2008) 93-103

32. Bagheri, E., Asadi, M., Gasevic, D., Soltani, S.: Stratified analytic hierarchy process: prioritization and selection of software features. In: Proceedings of the 14th international conference on Software product lines: going beyond. SPLC'10, Berlin, Heidelberg, Springer-Verlag (2010) 300-315

33. Janota, M., Kiniry, J.: Reasoning about feature models in higher-order logic. In: Proceedings of the 11th International Software Product Line Conference. SPLC '07, Washington, DC, USA, IEEE Computer Society (2007) 13-22

34. Benavides, D., Trinidad, P., Ruiz-Cortes, A.: Automated reasoning on feature models. In: LNCS, Advanced Information Systems Engineering: 17th International Conference, CAiSE 2005. Volume 3520., Springer (2005) 491-503

35. Batory, D.: Feature models, grammars, and propositional formulas. In: Proceedings of the 9th international conference on Software Product Lines. SPLC'05, Berlin, Heidelberg, Springer-Verlag (2005) 7-20

36. Boskovic, M., Bagheri, E., Gasevic, D., Mohabbati, B., Kaviani, N., Hatala, M.: Automated staged configuration with semantic web technologies. Int. J. Soft. Eng. Knowl. Eng. 20 (2010) 459-484

37. Bagheri, E., Di Noia, T., Ragone, A., Gasevic, D.: Configuring software product line feature models based on stakeholders' soft and hard requirements. In: Proceedings of the 14th international conference on Software product lines: going beyond. SPLC'10, Berlin, Heidelberg, Springer-Verlag (2010) 16-31

38. Benavides, D., Segura, S., Trinidad, P., Ruiz-Cortes, A.: FAMA: Tooling a framework for the automated analysis of feature models. In: Proceeding of the First International Workshop on Variability Modelling of Software-intensive Systems (VAMOS). (2007) 129-134

39. Bagheri, E., Noia, T.D., Gasevic, D., Ragone, A.: Formalizing interactive staged feature model configuration. Journal of Software: Evolution and Process 24 (2012) 375-400

40. Mendonca, M., Wasowski, A., Czarnecki, K., Cowan, D.: Efficient compilation techniques for large scale feature models. In: Proceedings of the 7 th international conference on Generative programming and component engineering, ACM New York, NY, USA (2008) 13-22

41. Wang, H., Li, Y., Sun, J., Zhang, H., Pan, J.: Verifying feature models using OWL. Web Semantics: Science, Services and Agents on the World Wide Web 5 (2007) 117-129

42. Perini, A., Ricca, F., Susi, A.: Tool-supported requirements prioritization: Comparing the ahp and cbrank methods. Information and Software Technology 51 (2009) 1021-1032

43. Aurum, A., Wohlin, C.: Engineering and managing software requirements. Springer-Verlag New York Inc (2005)

44. Lehtola, L., Kauppinen, M.: Suitability of requirements prioritization methods for marketdriven software product development. Software Process: Improvement and Practice 11 (2006) 7-19

45. Berander, P., Jonsson, P.: Hierarchical cumulative voting (hcv)-prioritization of requirements in hierarchies. International Journal of Software Engineering and Knowledge Engineering 16 (2006) 819-849 
46. Schmid, K., Rabiser, R., Grünbacher, P.: A comparison of decision modeling approaches in product lines. In: Proceedings of the 5th Workshop on Variability Modeling of SoftwareIntensive Systems, ACM (2011) 119-126

47. Dhungana, D., Grünbacher, P., Rabiser, R.: The dopler meta-tool for decision-oriented variability modeling: a multiple case study. Automated Software Engineering 18 (2011) $77-114$

48. Corporation, S.P.C.S.: Reuse-driven software processes. Technical Report SPC-92019-CMC, Version 02.00 .03 (1993)

49. Dhungana, D., Grübacher, P., Rabiser, R.: Decisionking: A flexible and extensible tool for integrated variability modeling. In: First International Workshop on Variability Modelling of Software-intensive Systems-Proceedings, Lero (2007) 119-127

50. Dhungana, D., Rabiser, R., Grunbacher, P.: Decision-oriented modeling of product line architectures. In: Proceedings of the Sixth Working IEEE/IFIP Conference on Software Architecture. WICSA '07, Washington, DC, USA, IEEE Computer Society (2007) 44-47 